Pacific

Journal of

Mathematics

\title{
GEOGRAPHY OF SIMPLY CONNECTED NONSPIN SYMPLECTIC 4-MANIFOLDS WITH POSITIVE SIGNATURE
}

Anar Akhmedov, Mark C. Hughes and B. Doug Park 


\title{
GEOGRAPHY OF SIMPLY CONNECTED NONSPIN SYMPLECTIC 4-MANIFOLDS WITH POSITIVE SIGNATURE
}

\author{
Anar Akhmedov, Mark C. Hughes and B. Doug Park
}

\begin{abstract}
We construct new families of closed simply connected nonspin irreducible symplectic 4-manifolds with positive signature that are interesting with respect to the geography problem.
\end{abstract}

\section{Introduction}

Given a closed smooth 4-manifold $M$, let $e(M)$ and $\sigma(M)$ denote the Euler characteristic and the signature of $M$, respectively. We define

$$
\chi_{h}(M)=\frac{e(M)+\sigma(M)}{4} \text { and } \quad c_{1}^{2}(M)=2 e(M)+3 \sigma(M) .
$$

Note that $e(M)$ and $\sigma(M)$ are in turn completely determined by $\chi_{h}(M)$ and $c_{1}^{2}(M)$, that is,

$$
e(M)=12 \chi_{h}(M)-c_{1}^{2}(M) \text { and } \quad \sigma(M)=c_{1}^{2}(M)-8 \chi_{h}(M) .
$$

When $M$ is a complex surface, $\chi_{h}(M)$ is the holomorphic Euler characteristic of $M$ while $c_{1}^{2}(M)$ is the square of the first Chern class of $M$. The classical "geography problem" in algebraic geometry, originally posed by Persson [1981], asks which ordered pairs of positive integers can be realized as the pair $\left(\chi_{h}(M), c_{1}^{2}(M)\right)$ for some minimal complex surface $M$ of general type. The related "botany problem", which is a lot more difficult, asks for the classification of all minimal complex surfaces with a given pair of invariants $\left(\chi_{h}, c_{1}^{2}\right)$.

The symplectic geography problem, first posed in [McCarthy and Wolfson 1994], asks which ordered pairs of integers can be realized as $\left(\chi_{h}(M), c_{1}^{2}(M)\right)$ for some minimal symplectic 4-manifold $M$. There has been steady progress on the symplectic geography problem in recent years and the problem has been completely solved for simply connected minimal symplectic 4-manifolds with negative signature

Akhmedov was partially supported by NSF grant DMS-1005741. Hughes was partially supported by an NSERC Canada graduate scholarship. Park was partially supported by an NSERC discovery grant. MSC2010: primary 57R17, 57R55; secondary 57M60, 57M12.

Keywords: symplectic 4-manifold, geography, branched covering, semifree action, generalized fiber sum. 
(cf. [Akhmedov et al. 2010a; Akhmedov and Park 2010a; Park and Szabó 2000]). The symplectic botany problem, that is, the classification problem for minimal symplectic 4-manifolds with a given pair of invariants $\left(\chi_{h}, c_{1}^{2}\right)$, seems to be an intractable problem at the moment. However, we now know that most ordered pairs are realized by infinitely many pairwise nondiffeomorphic simply connected minimal symplectic 4-manifolds; see [Gompf and Stipsicz 1999].

In this paper, we will focus our attention on the symplectic geography problem for simply connected minimal symplectic 4-manifolds with nonnegative signature. Unlike the negative signature case, the existing literature [Akhmedov and Park 2008; 2010b; Akhmedov et al. 2010b; Li and Stipsicz 2002; Niepel 2005; Park 2002; 2003; Stipsicz 1998; 1999] is far from capturing all possible $\left(\chi_{h}, c_{1}^{2}\right)$ coordinates, even if we allow nontrivial fundamental groups. The main goal of this paper is to summarize the current state of our knowledge when the simply connected symplectic 4-manifolds are required to be nonspin, or equivalently, are required to have odd intersection form. By Freedman's classification theorem [1982] for simply connected topological 4-manifolds, our problem is then equivalent to finding a minimal symplectic 4-manifold $M$ with signature $\sigma$ that is homeomorphic to $k \mathbb{C} \mathbb{P}^{2} \#(k-\sigma) \overline{\mathbb{C P}}^{2}$, where $k$ is any odd positive integer and $\sigma$ is any integer satisfying $0 \leq \sigma \leq k$. Here, $\mathbb{C P}^{2}$ is the complex projective plane, $\overline{\mathbb{C P}}^{2}$ is the underlying smooth 4-manifold $\mathbb{C P}^{2}$ equipped with the opposite orientation, and $k \mathbb{C P} \mathbb{P}^{2} \#(k-\sigma) \overline{\mathbb{C P}}^{2}$ is the connected sum of $k$ copies of $\mathbb{C P}^{2}$ and $k-\sigma$ copies of $\overline{\mathbb{C P}}^{2}$. Note that a simply connected symplectic 4-manifold $M$ has odd $b_{2}^{+}(M)$, and hence our integer $k$ must be odd.

A closed 4-manifold with signature $\sigma$ corresponds to a point $\left(\chi_{h}, c_{1}^{2}\right)$ on the line $c_{1}^{2}=8 \chi_{h}+\sigma$. For technical reasons, it will be convenient to fix the signature and deal with each of these lines separately. It is now well-known (see [Akhmedov and Park 2008; Park 2003]) that for each signature $\sigma \geq 0$, there exists a constant $\lambda(\sigma)$ depending only on $\sigma$ such that any point $\left(\chi_{h}, c_{1}^{2}\right)$ on the line $c_{1}^{2}=8 \chi_{h}+\sigma$ satisfying $\chi_{h} \geq \lambda(\sigma)$ is realized by at least one simply connected nonspin minimal symplectic 4-manifold and infinitely many simply connected nonspin irreducible nonsymplectic 4-manifolds (Definition 13 in Section 6). In other words, $k \mathbb{C P} \mathbb{P}^{2}(k-\sigma) \overline{\mathbb{C P}}^{2}$ is homeomorphic to at least one minimal symplectic 4-manifold and infinitely many pairwise nondiffeomorphic irreducible nonsymplectic 4-manifolds, provided that $k$ is odd and $k \geq 2 \lambda(\sigma)-1$ for some constant $\lambda(\sigma)$ that depends only on the signature $\sigma$.

The main result of this paper is the explicit formulation of the smallest values of $\lambda(\sigma)$ that are currently known to the authors. In [Akhmedov and Park 2008], small $\lambda(\sigma)$ values are given when $0 \leq \sigma \leq 4$, and these values are listed in Table 1 . In this paper, we will concentrate on the cases when $\sigma \geq 5$ (see Table 2 in Section 6). For example, when $0 \leq \sigma \leq 100$, we realize more than 20,000 new $\left(\chi_{h}, c_{1}^{2}\right)$ points that were not covered by the results in [Akhmedov and Park 2008; Park 2003]. 


\begin{tabular}{|c|c|c|c|c|c|}
\hline$\sigma$ & 0 & 1 & 2 & 3 & 4 \\
\hline$\lambda(\sigma) \leq$ & 25 & 25 & 24 & 27 & 26 \\
\hline
\end{tabular}

Table 1. Results from [Akhmedov and Park 2008].

If a 4-manifold $M$ is simply connected, then $2 \chi_{h}(M)-1=b_{2}^{+}(M) \geq \sigma(M)$. Thus we obtain an a priori lower bound $\chi_{h} \geq\lceil(\sigma+1) / 2\rceil$, where

$$
\lceil x\rceil=\min \{k \in \mathbb{Z} \mid k \geq x\}
$$

is the ceiling function. It is tempting to conjecture that our a posteriori lower bound for $\chi_{h}$ can eventually be improved down to $\lambda(\sigma)=\lceil(\sigma+1) / 2\rceil$, which will result in the complete solution of the geography problem for simply connected nonspin minimal symplectic 4-manifolds.

Our paper is organized as follows. In Section 2, we present a branched covering construction of Lefschetz fibrations with positive signature, which is a generalization of Stipsicz's constructions [1998; 1999]. In Section 3, we show how to glue together semifree cyclic group actions on closed 2-manifolds, and then we use these actions to construct new examples of Lefschetz fibrations with positive signature. In Section 4, we show how to obtain simply connected 4-manifolds from nonsimply connected Lefschetz fibrations by performing generalized fiber sums with certain 4-manifolds that were constructed in [Akhmedov and Park 2010a]. In Section 5, we implement the strategies from previous sections to construct new families of simply connected irreducible 4-manifolds with positive signature. In Section 6, we compute the lower bounds $\lambda(\sigma)$ for many small values of $\sigma$.

\section{Branched covering construction}

Let $\Sigma_{g}$ be a closed 2-dimensional manifold of genus $g>0$. Let $\zeta: \Sigma_{g} \rightarrow \Sigma_{g}$ be an orientation-preserving self-diffeomorphism of $\Sigma_{g}$ with $q$ fixed points $\left\{y_{1}, \ldots, y_{q}\right\}$. Assume that

$$
\zeta^{p}=\underbrace{\zeta \circ \cdots \circ \zeta}_{p}=\mathrm{id}
$$

for some positive integer $p \geq 2$, and that $\zeta$ generates a semifree $\mathbb{Z} / p$ action on $\Sigma_{g}$. If $\zeta_{*}: H_{1}\left(\Sigma_{g} ; \mathbb{Z}\right) \rightarrow H_{1}\left(\Sigma_{g} ; \mathbb{Z}\right)$ is the induced homomorphism on the first homology group, then we also assume that

$$
\zeta_{*}^{p-1}+\zeta_{*}^{p-2}+\cdots+\zeta_{*}+\mathrm{id}=0
$$

on $H_{1}\left(\Sigma_{g} ; \mathbb{Z}\right)$, which is equivalent to 1 not being an eigenvalue of $\zeta_{*}$. See Examples 3 and 5 below for some concrete examples of $\zeta$. 
We will consider $\Sigma_{g} \times \Sigma_{g}$ as a symplectic 4-manifold equipped with a product symplectic form $\tilde{\omega}=\operatorname{pr}_{1}^{*} \omega+\operatorname{pr}_{2}^{*} \omega$, where $\omega$ is a symplectic volume form on $\Sigma_{g}$ and $\operatorname{pr}_{j}: \Sigma_{g} \times \Sigma_{g} \rightarrow \Sigma_{g}(j=1,2)$ is the projection map onto the $j$-th factor. For each $i=1, \ldots, p$, let

$$
\Gamma_{i}=\operatorname{graph}\left(\zeta^{i}\right)=\left\{\left(x, \zeta^{i}(x)\right) \mid x \in \Sigma_{g}\right\} \subset \Sigma_{g} \times \Sigma_{g} .
$$

Note that $\Gamma_{p}$ is equal to the diagonal $\left\{(x, x) \mid x \in \Sigma_{g}\right\}$. The graphs $\Gamma_{1}, \ldots, \Gamma_{p}$ are symplectic submanifolds of $\Sigma_{g} \times \Sigma_{g}$ with respect to $\tilde{\omega}$ (see Lemma 2.1 in [Akhmedov and Park 2008]), and the graphs intersect at $q$ points

$$
\left\{\left(y_{j}, y_{j}\right) \mid j=1, \ldots, q\right\}
$$

If we symplectically blow up $\Sigma_{g} \times \Sigma_{g}$ at these $q$ intersection points, then the proper transform $B$ of the union $\Gamma_{1} \cup \cdots \cup \Gamma_{p}$ consists of $p$ disjoint genus $g$ symplectic submanifolds of $\left(\Sigma_{g} \times \Sigma_{g}\right) \# q \overline{\mathbb{C P}^{2}}$.

Let $\left\{\gamma_{k} \mid k=1, \ldots, 2 g\right\}$ be a basis for $H_{1}\left(\Sigma_{g} ; \mathbb{Z}\right)$ and let $\left\{\gamma^{\ell} \mid \ell=1, \ldots, 2 g\right\}$ be the dual basis under the intersection product so that $\gamma_{k} \cdot \gamma^{\ell}=\delta_{k}^{\ell}$. If we introduce the notation

$$
[\Delta]=\left[\Sigma_{g} \times\{\mathrm{pt}\}\right]+\left[\left\{\mathrm{pt}^{\prime}\right\} \times \Sigma_{g}\right],
$$

then the homology class of $\Gamma_{i}$ is given by

$$
\left[\Gamma_{i}\right]=[\Delta]-\sum_{k=1}^{2 g} \gamma^{k} \times \zeta_{*}^{i}\left(\gamma_{k}\right)
$$

Using (1), we can express the homology class of $B$ as

$$
[B]=p\left([\Delta]-\sum_{j=1}^{q}\left[E_{j}\right]\right),
$$

where $E_{1}, \ldots, E_{q}$ are the exceptional spheres of the blowups. We also note that

$$
c_{1}\left(\left(\Sigma_{g} \times \Sigma_{g}\right) \# q \overline{\mathbb{C P}}^{2}\right)=\operatorname{PD}\left((2-2 g)[\Delta]-\sum_{j=1}^{q}\left[E_{j}\right]\right),
$$

where PD denotes the Poincaré duality isomorphism.

Since $[B]$ is divisible by $p$, we may take the cyclic $p$-fold branched cover of $\left(\Sigma_{g} \times \Sigma_{g}\right) \# q \overline{\mathbb{C P}}^{2}$ that is branched along $B$. We will denote this branched covering by $\beta: X_{g, p, q}^{\zeta} \rightarrow\left(\Sigma_{g} \times \Sigma_{g}\right) \# q \overline{\mathbb{C P}}^{2}$. The total space $X_{g, p, q}^{\zeta}$ inherits a symplectic 
structure from $\left(\Sigma_{g} \times \Sigma_{g}\right) \# q \overline{\mathbb{C P}}^{2}$, and we have

$$
\begin{aligned}
c_{1}\left(X_{g, p, q}^{\zeta}\right) & =\beta^{*}\left(c_{1}\left(\left(\Sigma_{g} \times \Sigma_{g}\right) \# q \overline{\mathbb{C P}}^{2}\right)-\frac{p-1}{p} \operatorname{PD}[B]\right) \\
& =\beta^{*} \operatorname{PD}\left((3-2 g-p)[\Delta]+(p-2) \sum_{j=1}^{q}\left[E_{j}\right]\right) .
\end{aligned}
$$

The characteristic numbers of $X_{g, p, q}^{\zeta}$ can be computed as follows.

$$
\begin{aligned}
e\left(X_{g, p, q}^{\zeta}\right) & =p e\left(\left(\Sigma_{g} \times \Sigma_{g}\right) \# q \overline{\mathbb{C P}}^{2}\right)-p(p-1) e\left(\Sigma_{g}\right) \\
& =p\left((2-2 g)^{2}+q\right)-p(p-1)(2-2 g) \\
& =p\left(4 g^{2}+2 g p-10 g-2 p+q+6\right) \\
c_{1}^{2}\left(X_{g, p, q}^{\zeta}\right) & =p\left((3-2 g-p)[\Delta]+(p-2) \sum_{j=1}^{q}\left[E_{j}\right]\right)^{2} \\
& =p\left(2(3-2 g-p)^{2}-q(p-2)^{2}\right) \\
& =p\left(-p^{2} q+8 g^{2}+2 p^{2}+8 g p+4 p q-24 g-12 p-4 q+18\right), \\
\sigma\left(X_{g, p, q}^{\zeta}\right) & =\frac{1}{3}\left(c_{1}^{2}\left(X_{g, p, q}^{\zeta}\right)-2 e\left(X_{g, p, q}^{\zeta}\right)\right) \\
& =\frac{1}{3} p\left(-p^{2} q+2 p^{2}+4 g p+4 p q-4 g-8 p-6 q+6\right), \\
\chi_{h}\left(X_{g, p, q}^{\zeta}\right) & =\frac{1}{4}\left(e\left(X_{g, p, q}^{\zeta}\right)+\sigma\left(X_{g, p, q}^{\zeta}\right)\right) \\
& =\frac{1}{12} p\left(-p^{2} q+12 g^{2}+2 p^{2}+10 g p+4 p q-34 g-14 p-3 q+24\right) .
\end{aligned}
$$

Let $\epsilon:\left(\Sigma_{g} \times \Sigma_{g}\right) \# q \overline{\mathbb{C P}}^{2} \rightarrow \Sigma_{g} \times \Sigma_{g}$ be the blowdown map. Then the composition of maps

$$
X_{g, p, q}^{\zeta} \stackrel{\beta}{\longrightarrow}\left(\Sigma_{g} \times \Sigma_{g}\right) \# q \overline{\mathbb{C P}^{2}} \stackrel{\epsilon}{\longrightarrow} \Sigma_{g} \times \Sigma_{g} \stackrel{\mathrm{pr}_{1}}{\longrightarrow} \Sigma_{g}
$$

gives a fibration of $X_{g, p, q}^{\zeta}$ over $\Sigma_{g}$. A regular fiber of this fibration is a cyclic $p$-fold branched cover of $\Sigma_{g}$ that is branched over $p$ points. Thus a regular fiber is a closed surface of genus equal to

$$
\frac{1}{2}\left(p^{2}+2 g p-3 p+2\right) .
$$

The proper transform of each graph $\Gamma_{i}(i=1, \ldots, p)$ gives rise to a section of (2) whose image is a genus $g$ surface $S_{i}$ in $X_{g, p, q}^{\zeta}$ with self-intersection equal to

$$
\begin{aligned}
{\left[S_{i}\right]^{2} } & =\left\langle c_{1}\left(X_{g, p, q}^{\zeta}\right),\left[S_{i}\right]\right\rangle-e\left(\Sigma_{g}\right) \\
& =2 g-2+\frac{1}{p}\left((3-2 g-p)[\Delta]+(p-2) \sum_{j=1}^{q}\left[E_{j}\right]\right) \cdot[B] \\
& =p q-2 g-2 p-2 q+4 .
\end{aligned}
$$


Lemma 1. Let $f: X_{g, p, q}^{\zeta} \rightarrow \Sigma_{g}$ denote the composition of maps in (2). Then $f$ is a relatively minimal Lefschetz fibration with pq critical points. Moreover, each critical point of $f$ corresponds to a nonseparating vanishing cycle.

Proof. Clearly the only singular fibers of $f$ are $\left\{f^{-1}\left(y_{j}\right) \mid j=1, \ldots, q\right\}$. We will prove that each $f^{-1}\left(y_{j}\right)$ contains exactly $p$ Lefschetz critical points. To describe each $f^{-1}\left(y_{j}\right)$ explicitly, we will view $X_{g, p, q}^{\zeta}$ as the minimal desingularization of another branched cover that we will define below.

Let $\Gamma=\Gamma_{1} \cup \cdots \cup \Gamma_{p}$. Since $[\Gamma]=p[\Delta] \in H_{2}\left(\Sigma_{g} \times \Sigma_{g} ; \mathbb{Z}\right)$ is divisible by $p$, we may take the cyclic $p$-fold branched cover of $\Sigma_{g} \times \Sigma_{g}$ that is branched along $\Gamma$. We will denote this branched covering by $\widehat{\beta}: \widehat{X}_{g, p, q}^{\zeta} \rightarrow \Sigma_{g} \times \Sigma_{g}$. The total space $\widehat{X}_{g, p, q}^{\zeta}$ has $q$ singular points, $\left\{\widehat{\beta}^{-1}\left(y_{j}, y_{j}\right) \mid j=1, \ldots, q\right\}$, each of which can be locally modeled by

$$
\left\{(x, y, z) \in \mathbb{C}^{3} \mid z^{p}=x^{p}+y^{p}\right\} .
$$

In these local coordinates, the singular point $\widehat{\beta}^{-1}\left(y_{j}, y_{j}\right)$ corresponds to $(0,0,0)$, and a neighborhood of the singular point corresponds to the cyclic $p$-fold cover of the $(x, y)$-plane that is branched over $p$ complex lines that intersect transversely at $(0,0)$.

Next let $\widehat{f}: \widehat{X}_{g, p, q}^{\zeta} \rightarrow \Sigma_{g}$ denote the singular fibration given by the composition

$$
\widehat{X}_{g, p, q}^{\zeta} \stackrel{\widehat{\beta}}{\longrightarrow} \Sigma_{g} \times \Sigma_{g} \stackrel{\mathrm{pr}_{1}}{\longrightarrow} \Sigma_{g} .
$$

A regular fiber of $\widehat{f}$ is again a closed surface of genus equal to (3). There are exactly $q$ singular fibers $\left\{\widehat{f}^{-1}\left(y_{j}\right) \mid j=1, \ldots, q\right\}$. For each $j=1, \ldots, q$, note that $\widehat{f}^{-1}\left(y_{j}\right) \backslash\left\{\widehat{\beta}^{-1}\left(y_{j}, y_{j}\right)\right\}$ is a smooth and connected surface since it is the unbranched cyclic $p$-fold cover of the once punctured surface $\left(\left\{y_{j}\right\} \times \Sigma_{g}\right) \backslash\left\{\left(y_{j}, y_{j}\right)\right\}$ coming from a surjective homomorphism

$$
\pi_{1}\left(\left(\left\{y_{j}\right\} \times \Sigma_{g}\right) \backslash\left\{\left(y_{j}, y_{j}\right)\right\}\right) \cong F_{2 g} \longrightarrow \mathbb{Z} / p \subset S_{p},
$$

where $F_{2 g}$ is the free group with $2 g$ generators and $S_{p}$ is the symmetric group on $p$ symbols. Since $\mathbb{Z} / p$ is abelian, (5) can be factored as the composition

$$
\pi_{1}\left(\left(\left\{y_{j}\right\} \times \Sigma_{g}\right) \backslash\left\{\left(y_{j}, y_{j}\right)\right\}\right) \longrightarrow \pi_{1}\left(\Sigma_{g}\right) \longrightarrow \mathbb{Z} / p .
$$

Thus the cover $\widehat{f}^{-1}\left(y_{j}\right) \backslash\left\{\widehat{\beta}^{-1}\left(y_{j}, y_{j}\right)\right\} \rightarrow\left(\left\{y_{j}\right\} \times \Sigma_{g}\right) \backslash\left\{\left(y_{j}, y_{j}\right)\right\}$ can be viewed as a restriction of the unbranched cyclic $p$-fold cover of the closed surface $\Sigma_{g}$. In other words, $\widehat{f}^{-1}\left(y_{j}\right) \backslash\left\{\widehat{\beta}^{-1}\left(y_{j}, y_{j}\right)\right\}$ can be embedded into the unbranched cyclic $p$-fold cover of $\Sigma_{g}$. This implies that $\widehat{f}^{-1}\left(y_{j}\right) \backslash\left\{\widehat{\beta}^{-1}\left(y_{j}, y_{j}\right)\right\}$ is diffeomorphic to a surface of genus $g p-p+1$ having $p$ punctures, and $\hat{f}^{-1}\left(y_{j}\right)$ is a connected surface that is smooth away from the point $\widehat{\beta}^{-1}\left(y_{j}, y_{j}\right)$, which is a multiple point of order $p$. 
Now recall from [Gompf and Stipsicz 1999; Némethi 1999] that $X_{g, p, q}^{\zeta}$ is the minimal desingularization of $\widehat{X}_{g, p, q}^{\zeta}$. The standard algorithm for resolution of singularities (see [Némethi 1999, Example 1.20(h)]) replaces each singular point $\widehat{\beta}^{-1}\left(y_{j}, y_{j}\right)$ of $\widehat{X}_{g, p, q}^{\zeta}$ having local model (4) with a closed surface of genus $\frac{1}{2}\left(p^{2}-3 p+2\right)$ and self-intersection $-p$. This surface is just $\beta^{-1}\left(E_{j}\right)$, which is a cyclic $p$-fold branched cover of the exceptional sphere $E_{j}$ branched over $p$ points. It follows that each singular fiber $f^{-1}\left(y_{j}\right)$ is the union of two closed surfaces that intersect each other transversely at $p$ distinct points. One of the surfaces is $\beta^{-1}\left(E_{j}\right)$, and the other is a genus $g p-p+1$ surface of self-intersection $-p$, which is the smooth completion of $\widehat{f}^{-1}\left(y_{j}\right) \backslash\left\{\widehat{\beta}^{-1}\left(y_{j}, y_{j}\right)\right\}$. The $p$ transverse intersection points between the two surfaces are exactly the $p$ Lefschetz critical points of $f$ that get mapped to $y_{j}$. Finally, comparing the sum of genera with (3), we observe that each union of the two surfaces is obtained by replacing the annular neighborhoods of $p$ nonseparating circles in a regular fiber with $p$ pairs of transversely intersecting disks. This implies that all the vanishing cycles are nonseparating.

Remark 2. We can verify the number of critical points of $f$ by computing the difference

$$
e\left(X_{g, p, q}^{\zeta}\right)-e(\text { regular fiber }) \cdot e(\text { base })=p q .
$$

We can split the singular fibers of $f$ so that each new singular fiber contains only one critical point (cf. [Harris and Morrison 1998; Takamura 2004]) but we do not need to do so for our applications below.

Given a positive integer $u$, let $\eta_{u}: \Sigma_{k} \rightarrow \Sigma_{g}$ be a $u$-fold unbranched covering of $\Sigma_{g}$, where $k=u(g-1)+1$. We pull back the branched covering

$$
X_{g, p, q}^{\zeta} \stackrel{\beta}{\longrightarrow}\left(\Sigma_{g} \times \Sigma_{g}\right) \# q \overline{\mathbb{C P}}^{2} \stackrel{\epsilon}{\longrightarrow} \Sigma_{g} \times \Sigma_{g}
$$

by the product map $\eta_{u_{1}} \times \eta_{u_{2}}: \Sigma_{k_{1}} \times \Sigma_{k_{2}} \rightarrow \Sigma_{g} \times \Sigma_{g}$, where $u_{i}$ is a positive integer and $k_{i}=u_{i}(g-1)+1$ for each $i=1,2$. The total space of this pullback is a new symplectic 4-manifold $X_{g, p, q}^{\zeta}\left(u_{1}, u_{2}\right)$, which is a $p$-fold branched cover of $\Sigma_{k_{1}} \times \Sigma_{k_{2}}$ and a $u_{1} u_{2}$-fold unbranched cover of $X_{g, p, q}^{\zeta}$. The composition

$$
f_{u_{1}, u_{2}}: X_{g, p, q}^{\zeta}\left(u_{1}, u_{2}\right) \longrightarrow \Sigma_{k_{1}} \times \Sigma_{k_{2}} \stackrel{\mathrm{pr}_{1}}{\longrightarrow} \Sigma_{k_{1}}
$$

gives a new relatively minimal Lefschetz fibration, where $X_{g, p, q}^{\zeta}(1,1)=X_{g, p, q}^{\zeta}$ and $f_{1,1}=f$. A regular fiber of $f_{u_{1}, u_{2}}$ is a $u_{2}$-fold unbranched cover of the fiber of $f$ (or equivalently a $p$-fold branched cover of $\Sigma_{k_{2}}$ branched along $u_{2} p$ points) and hence has genus equal to

$$
1+\frac{u_{2}}{2}\left(p^{2}+2 g p-3 p\right)
$$


A section of $f$ gives rise to a section of $f_{u_{1}, u_{2}}$ whose image is a genus $k_{1}$ surface of self-intersection equal to

$$
u_{1}(p q-2 g-2 p-2 q+4) .
$$

Since $X_{g, p, q}^{\zeta}\left(u_{1}, u_{2}\right)$ is a $u_{1} u_{2}$-fold unbranched cover of $X_{g, p, q}^{\zeta}$, we have

$$
\begin{array}{cc}
e\left(X_{g, p, q}^{\zeta}\left(u_{1}, u_{2}\right)\right)=u_{1} u_{2} \cdot e\left(X_{g, p, q}^{\zeta}\right), & \sigma\left(X_{g, p, q}^{\zeta}\left(u_{1}, u_{2}\right)\right)=u_{1} u_{2} \cdot \sigma\left(X_{g, p, q}^{\zeta}\right), \\
\chi_{h}\left(X_{g, p, q}^{\zeta}\left(u_{1}, u_{2}\right)\right)=u_{1} u_{2} \cdot \chi_{h}\left(X_{g, p, q}^{\zeta}\right), & c_{1}^{2}\left(X_{g, p, q}^{\zeta}\left(u_{1}, u_{2}\right)\right)=u_{1} u_{2} \cdot c_{1}^{2}\left(X_{g, p, q}^{\zeta}\right) .
\end{array}
$$

Example 3. Recall from Section 2 of [Akhmedov and Park 2008] that there exists a semifree $\mathbb{Z} /(g+1)$ action on $\Sigma_{g}$ with 4 fixed points satisfying (1). Applying the above machinery, we obtain a family of symplectic 4-manifolds $X_{u_{1}, u_{2}}^{g}=$ $X_{g, g+1,4}^{\zeta}\left(u_{1}, u_{2}\right)$, where $g, u_{1}$ and $u_{2}$ are positive integers, satisfying

$$
\begin{aligned}
e\left(X_{u_{1}, u_{2}}^{g}\right) & =2 u_{1} u_{2}(g+1)\left(3 g^{2}-5 g+4\right), \\
\sigma\left(X_{u_{1}, u_{2}}^{g}\right) & =\frac{2}{3} u_{1} u_{2}(g+1)\left(g^{2}+2 g-6\right), \\
\chi_{h}\left(X_{u_{1}, u_{2}}^{g}\right) & =\frac{1}{6} u_{1} u_{2}(g+1)\left(10 g^{2}-13 g+6\right), \\
c_{1}^{2}\left(X_{u_{1}, u_{2}}^{g}\right) & =2 u_{1} u_{2}(g+1)\left(7 g^{2}-8 g+2\right) .
\end{aligned}
$$

For each triple of positive integers $g, u_{1}, u_{2}$, there exists a relatively minimal Lefschetz fibration $f_{u_{1}, u_{2}}: X_{u_{1}, u_{2}}^{g} \rightarrow \Sigma_{k_{1}}$ such that the genus of a regular fiber is equal to $1+\frac{1}{2} u_{2}(g+1)(3 g-2)$ and there is a section whose image is a surface of genus $k_{1}=u_{1}(g-1)+1$ and self-intersection $-2 u_{1}$.

Remark 4. The 4-manifolds $X_{g}, X_{g}(n)$ and $\tilde{X}_{g}\left(n^{2}\right)$ in [Akhmedov and Park 2008] are equal to $X_{1,1}^{g}, X_{n, 1}^{g}$ and $X_{n, n}^{g}$, respectively.

\section{Gluing self-diffeomorphisms of surfaces}

In light of the machinery in Section 2, it will be desirable to find lots of semifree $\mathbb{Z} / p$ actions on closed surfaces. One way to produce such actions is to glue together semifree $\mathbb{Z} / p$ actions on surfaces of low genera as we explain below.

Let $v \geq 2$ be an integer. For each $i=1, \ldots, v$, let $\alpha_{i}: \Sigma_{g_{i}} \rightarrow \Sigma_{g_{i}}$ be an orientationpreserving self-diffeomorphism of a closed surface of genus $g_{i}$ with $q_{i}$ fixed points $\left\{y_{i, 1}, \ldots, y_{i, q_{i}}\right\}$. Assume that each $\alpha_{i}$ generates a semifree $\mathbb{Z} / p$ action on $\Sigma_{g_{i}}$. For each $j=1, \ldots, q_{i}$, let $\rho_{i, j}$ be the rotational number of $\alpha_{i}$ at the fixed point $y_{i, j}$ so that $\alpha_{i}$ induces rotation by angle $2 \pi \rho_{i, j} / p$ in the tangent space at $y_{i, j}$. The rotational numbers are well-defined $\bmod p$ and are relatively prime to $p$. They satisfy (see [Nielsen 1937])

$$
\sum_{j=1}^{q_{i}} \frac{1}{\rho_{i, j}} \equiv 0(\bmod p),
$$


where $1 / \rho_{i, j}$ denotes the multiplicative inverse of $\rho_{i, j}$ in $(\mathbb{Z} / p)^{\times}$. We can reverse the signs of $\rho_{i, 1}, \ldots, \rho_{i, q_{i}}$ simultaneously by reversing the orientation of $\Sigma_{g_{i}}$.

Now choose a single fixed point of $\alpha_{i}$ for $i=1, v$, and choose two fixed points of $\alpha_{i}$ for $i=2, \ldots, v-1$. Without loss of generality, we may choose $y_{1,2}, y_{v, 1}$ and $y_{i, 1}, y_{i, 2}$ for $i=2, \ldots, v-1$. We remove small $\mathbb{Z} / p$-equivariant neighborhoods of these chosen fixed points and then glue the boundary circle at $y_{i, 2}$ to the boundary circle at $y_{i+1,1}$ for $i=1, \ldots, v-1$. Such gluing of one-holed and two-holed surfaces results in a closed surface of genus $g=\sum_{i=1}^{v} g_{i}$. If $\rho_{i, 2}=-\rho_{i+1,1}$ for all $i=1, \ldots, v-1$, that is, the rotational numbers are negatives of each other at the gluing points, then the restrictions of $\alpha_{i}$ 's to the punctured surfaces can also be glued together to form an orientation-preserving self-diffeomorphism $\zeta: \Sigma_{g} \rightarrow \Sigma_{g}$ with $q$ fixed points, where

$$
q=-2(v-1)+\sum_{i=1}^{v} q_{i} .
$$

We will say that $\zeta$ is an equivariant sum of $\alpha_{1}, \ldots, \alpha_{v}$, and write $\zeta=\alpha_{1} \# \ldots \# \alpha_{v}$. In case when $\alpha_{1}=\cdots=\alpha_{v}$, we will write $\zeta=v \alpha_{1}$ for short.

Example 5. For each odd integer $p \geq 3$, there exists a semifree $\mathbb{Z} / p$ action on $\Sigma_{(p-1) / 2}$ as follows. Consider $\Sigma_{(p-1) / 2}$ as the quotient of a regular $2 p$-gon by identifying the opposite sides. The rotation of the $2 p$-gon by angle $2 \pi / p$ gives an orientation-preserving self-diffeomorphism $\tau_{p}: \Sigma_{(p-1) / 2} \rightarrow \Sigma_{(p-1) / 2}$ with 3 fixed points. The fixed points of $\tau_{p}$ are the center of the $2 p$-gon and the 2 points coming from the vertices. The center of the $2 p$-gon has rotational number 1 , and the other 2 fixed points both have rotational number -2 .

We can find a basis of $H_{1}\left(\Sigma_{(p-1) / 2} ; \mathbb{Z}\right)$ such that the induced homomorphism $\left(\tau_{p}\right)_{*}: H_{1}\left(\Sigma_{(p-1) / 2} ; \mathbb{Z}\right) \rightarrow H_{1}\left(\Sigma_{(p-1) / 2} ; \mathbb{Z}\right)$ is represented by the $(p-1) \times(p-1)$ matrix

$$
\left[\begin{array}{rrr|r}
0 & \cdots & 0 & -1 \\
\hline & & & -1 \\
& & & \\
& I_{p-2} & & \vdots \\
& & & -1
\end{array}\right],
$$

where $I_{p-2}$ is the identity $(p-2) \times(p-2)$ matrix. It is easy to check that this matrix satisfies (1).

For each positive integer $v$, let $\zeta=v \tau_{p}$ be the equivariant sum of $v$ copies of $\tau_{p}$. (We glue along fixed points with rotational number -2 , and we alternate the orientations of the punctured $\Sigma_{(p-1) / 2}$ 's so that the rotational numbers are +2 and -2 at each gluing.) Then $\zeta: \Sigma_{v(p-1) / 2} \rightarrow \Sigma_{v(p-1) / 2}$ generates a semifree $\mathbb{Z} / p$ action on $\Sigma_{v(p-1) / 2}$ with $v+2$ fixed points. The induced homomorphism 
$\zeta_{*}: H_{1}\left(\Sigma_{v(p-1) / 2} ; \mathbb{Z}\right) \rightarrow H_{1}\left(\Sigma_{v(p-1) / 2} ; \mathbb{Z}\right)$ satisfies $(1)$ since it can be represented by a block diagonal matrix each of whose blocks is conjugate to (6).

From the branched covering construction in Section 2, we obtain a family of symplectic 4-manifolds $W_{u_{1}, u_{2}}^{p, v}=X_{v(p-1) / 2, p, v+2}^{v \tau_{p}}\left(u_{1}, u_{2}\right)$, where $p \geq 3$ is an odd integer and $v, u_{1}, u_{2}$ are positive integers, satisfying

$$
\begin{aligned}
e\left(W_{u_{1}, u_{2}}^{p, v}\right) & =p u_{1} u_{2}\left[\left(v^{2}+v\right) p^{2}-2\left(v^{2}+3 v+1\right) p+v^{2}+6 v+8\right], \\
\sigma\left(W_{u_{1}, u_{2}}^{p, v}\right) & =\frac{1}{3} p u_{1} u_{2}\left(v p^{2}-4 v-6\right), \\
\chi_{h}\left(W_{u_{1}, u_{2}}^{p, v}\right) & =\frac{1}{12} p u_{1} u_{2}\left[\left(3 v^{2}+4 v\right) p^{2}-6\left(v^{2}+3 v+1\right) p+3 v^{2}+14 v+18\right], \\
c_{1}^{2}\left(W_{u_{1}, u_{2}}^{p, v}\right) & =p u_{1} u_{2}\left[\left(2 v^{2}+3 v\right) p^{2}-4\left(v^{2}+3 v+1\right) p+2 v^{2}+8 v+10\right] .
\end{aligned}
$$

Moreover, for each quadruple of positive integers $p, v, u_{1}, u_{2}$ with odd $p \geq 3$, we have a relatively minimal Lefschetz fibration $f_{u_{1}, u_{2}}: W_{u_{1}, u_{2}}^{p, v} \rightarrow \Sigma_{k_{1}}$ such that the genus of a regular fiber is equal to $1+\frac{1}{2} p u_{2}[(v+1) p-v-3]$ and there is a section whose image is a surface of genus $k_{1}=1+u_{1}[-1+v(p-1) / 2]$ and self-intersection $-u_{1} v$.

Note that $c_{1}^{2}\left(W_{u_{1}, u_{2}}^{p, v}\right) \leq 9 \chi_{h}\left(W_{u_{1}, u_{2}}^{p, v}\right)$, with equality if and only if $p=5$ and $v=1$. If we view the quotient $c_{1}^{2}\left(W_{u_{1}, u_{2}}^{p, v}\right) / \chi_{h}\left(W_{u_{1}, u_{2}}^{p, v}\right)$ as a function of $p$ and $v$, then its gradient vector is

$$
\left[\begin{array}{c}
-\frac{24\left(\left(v^{3}+3 v^{2}+v\right) p^{2}-\left(5 v^{3}+16 v^{2}+14 v\right) p+4 v^{3}+18 v^{2}+22 v+6\right)}{\left(\left(3 v^{2}+4 v\right) p^{2}-6\left(v^{2}+3 v+1\right) p+3 v^{2}+14 v+18\right)^{2}} \\
-\frac{12\left(\left(p^{2}-4\right)(p-1)^{2} v^{2}-12(p-1)^{2} v+2 p^{3}-14 p^{2}+28 p-4\right)}{\left(\left(3 v^{2}+4 v\right) p^{2}-6\left(v^{2}+3 v+1\right) p+3 v^{2}+14 v+18\right)^{2}}
\end{array}\right]
$$

When $p \geq 7$ and $v \geq 1$, both components of this gradient vector are negative and hence $c_{1}^{2}\left(W_{u_{1}, u_{2}}^{p, v}\right) / \chi_{h}\left(W_{u_{1}, u_{2}}^{p, v}\right)$ is decreasing as $p$ and $v$ increase. We observe that $\lim _{v \rightarrow \infty} c_{1}^{2}\left(W_{u_{1}, u_{2}}^{p, v}\right) / \chi_{h}\left(W_{u_{1}, u_{2}}^{p, v}\right)=8$, and

$$
\lim _{p \rightarrow \infty} \frac{c_{1}^{2}\left(W_{u_{1}, u_{2}}^{p, v}\right)}{\chi_{h}\left(W_{u_{1}, u_{2}}^{p, v}\right)}=\frac{12(2 v+3)}{3 v+4} \leq \frac{60}{7},
$$

where the rational function $12(2 v+3) /(3 v+4)$ is decreasing for $v \geq 1$. Therefore most $W_{u_{1}, u_{2}}^{p, v}$ 's lie well below the Bogomolov-Miyaoka-Yau (BMY) line, $c_{1}^{2}=9 \chi_{h}$.

Remark 6. According to Section 4.5 of [Luo 2000], there is a unique $\mathbb{Z} / 3$ action on $\Sigma_{g}$ with $g+2$ fixed points. It follows that $W_{u_{1}, u_{2}}^{3,2}$ is exactly equal to $X_{u_{1}, u_{2}}^{2}$ in Example 3. More generally, for each odd integer $p \geq 5$, we conjecture that $W_{u_{1}, u_{2}}^{p, 2}$ is diffeomorphic to $X_{u_{1}, u_{2}}^{p-1}$ in Example 3. We also conjecture that the 4-manifolds $Z_{g}$, $Z_{g}(n)$ and $\tilde{Z}_{g}\left(n^{2}\right)$ in Section 3 of [Akhmedov and Park 2008] are diffeomorphic to $W_{1,1}^{2 g+1,1}, W_{n, 1}^{2 g+1,1}$ and $W_{n, n}^{2 g+1,1}$, respectively. In particular, we conjecture that 
$W_{1,1}^{5,1}, W_{n, 1}^{5,1}$ and $W_{n, n}^{5,1}$, lying on the BMY line $c_{1}^{2}=9 \chi_{h}$, are diffeomorphic to complex surfaces $H=H(1), H(n)$ and $H\left(n^{2}\right)$ in [Chen 1991; Stipsicz 1998; 1999], respectively.

\section{Generalized fiber sums}

Let $\Sigma_{b}$ denote a closed Riemann surface of genus $b>0$. Suppose $f: X \rightarrow \Sigma_{b}$ is a Lefschetz fibration with generic fiber $F$ diffeomorphic to a closed Riemann surface $\Sigma_{a}$ with genus $a>0$. Assume that $f$ is a relatively minimal Lefschetz fibration (i.e., no fiber contains a sphere of self-intersection -1 ) so that $X$ is a minimal symplectic 4-manifold (Theorem 1.4 of [Stipsicz 2000]). Also assume that $f$ has a section whose image $S$ in $X$ has self-intersection $d$. From Theorem 10.2.18 in [Gompf and Stipsicz 1999], $X$ can be equipped with a symplectic structure such that both $F$ and $S$ are symplectic submanifolds. From Proposition 8.1.9 in [Gompf and Stipsicz 1999], we have an exact sequence

$$
\pi_{1}(F) \longrightarrow \pi_{1}(X) \stackrel{f_{*}}{\longrightarrow} \pi_{1}\left(\Sigma_{b}\right) \longrightarrow 1 .
$$

Let $t>0$ be an integer. By symplectically resolving the intersection points, we can find a symplectic genus $t a+b$ surface $\Sigma \subset X$ representing the homology class $t[F]+[S] \in H_{2}(X ; \mathbb{Z})$ with self-intersection $2 t+d$. By taking $t$ large enough, we can assume that $2 t+d \geq 0$. Let $\tilde{X}=X \#(2 t+d) \overline{\mathbb{C P}}^{2}$, where each of the $2 t+d$ symplectic blowups take place at points on $\Sigma \subset X$. The proper transform $\widetilde{\Sigma} \subset \widetilde{X}$ is a symplectic submanifold with genus $t a+b$ and self-intersection 0 . Note that we have

$$
\begin{aligned}
e(\tilde{X}) & =e(X)+2 t+d, \\
\sigma(\tilde{X}) & =\sigma(X)-2 t-d .
\end{aligned}
$$

Lemma 7. Let $\tilde{i}: \widetilde{\Sigma}^{\|} \hookrightarrow \widetilde{X} \backslash v \widetilde{\Sigma}$ be the inclusion map of a parallel copy of $\widetilde{\Sigma}$ into the complement of a tubular neighborhood $v \widetilde{\Sigma}$ in $\widetilde{X}=X \#(2 t+d) \overline{\mathbb{C P}}^{2}$. Then we have

$$
\frac{\pi_{1}(\tilde{X} \backslash v \widetilde{\Sigma})}{\left\langle\tilde{i}_{*}\left(\pi_{1}(\widetilde{\Sigma} \|)\right)\right\rangle}=1,
$$

where $\left\langle\tilde{i}_{*}\left(\pi_{1}\left(\widetilde{\Sigma}^{\|}\right)\right)\right\rangle$is the normal subgroup of $\pi_{1}(\widetilde{X} \backslash v \widetilde{\Sigma})$ generated by the image $\tilde{i}_{*}\left(\pi_{1}\left(\widetilde{\Sigma}^{\|}\right)\right)$.

Proof. Let $i: \Sigma^{\|} \hookrightarrow X \backslash \nu \Sigma$ be the inclusion map of a parallel copy of $\Sigma$. From exact sequence (7), we deduce that $\pi_{1}(X) /\left\langle i_{*}\left(\pi_{1}\left(\Sigma^{\|}\right)\right)\right\rangle=1$. Since the blowups do not effect the fundamental groups, we conclude that $\pi_{1}(\widetilde{X}) /\left\langle\tilde{i}_{*}\left(\pi_{1}\left(\widetilde{\Sigma}^{\|}\right)\right)\right\rangle=1$. If 
$2 t+d>0$, then any meridian $\mu(\widetilde{\Sigma})$ of $\widetilde{\Sigma}$ in $\pi_{1}(\widetilde{X} \backslash v \widetilde{\Sigma})$ bounds a disk that comes from a punctured exceptional sphere. Hence $\pi_{1}(\widetilde{X} \backslash v \widetilde{\Sigma})=\pi_{1}(\widetilde{X})$ and (8) follows from our last conclusion.

If $2 t+d=0$, then $\widetilde{X}=X, \widetilde{\Sigma}=\Sigma, \widetilde{\Sigma}^{\|}=\Sigma^{\|}$, and $\tilde{i}=i$. Any meridian $\mu(\Sigma)$ in $\pi_{1}(X \backslash v \Sigma)$ is conjugate to a meridian of $S$. Since $[F] \cdot[S]=1, \mu(\Sigma)$ is in the normal subgroup generated by the generators of $\pi_{1}(F)$, which in turn lies in $\left\langle i_{*}\left(\pi_{1}\left(\Sigma^{\|}\right)\right)\right\rangle$. This implies that $\pi_{1}(X \backslash v \Sigma) /\left\langle i_{*}\left(\pi_{1}\left(\Sigma^{\|}\right)\right)\right\rangle=\pi_{1}(X) /\left\langle i_{*}\left(\pi_{1}\left(\Sigma^{\|}\right)\right)\right\rangle=1$.

For each pair of integers $m \geq 1$ and $n \geq 2$, let $Y_{n}(m)$ denote the irreducible 4-manifold constructed in Section 2 of [Akhmedov and Park 2010a] that has the same cohomology ring as the connected sum $(2 n-3)\left(S^{2} \times S^{2}\right)$. Recall that $Y_{n}(m)$ is obtained by performing $2 n+4$ surgeries along Lagrangian tori in the product 4-manifold $\Sigma_{2} \times \Sigma_{n}$. Thus $Y_{n}(m)$ contains a pair of submanifolds $\Sigma_{2}=\Sigma_{2} \times$ \{pt $\}$ and $\Sigma_{n}=\left\{\mathrm{pt}^{\prime}\right\} \times \Sigma_{n}$, both of self-intersection 0 . When $m=1, Y_{n}(1)$ is a minimal symplectic 4-manifold. Moreover, $\Sigma_{2}$ and $\Sigma_{n}$ are symplectic submanifolds of $Y_{n}(1)$. When $n \geq 3$, there exist $2 n-4$ pairs of geometrically dual Lagrangian tori which, together with $\Sigma_{2}$ and $\Sigma_{n}$, form a basis for $H_{2}\left(Y_{n}(1) ; \mathbb{Z}\right) \cong \mathbb{Z}^{4 n-6}$.

Theorem 8. Let $f: X \rightarrow \Sigma_{b}$ be a relatively minimal Lefschetz fibration as above having at least one nonseparating vanishing cycle. Suppose that $n=t a+b \geq 2$. For a suitable choice of the gluing diffeomorphism $\varphi: \partial(\nu \widetilde{\Sigma}) \rightarrow \partial\left(\nu \Sigma_{n}\right)$, the generalized fiber sum

$$
P_{n}^{m}(X)=\widetilde{X} \# \varphi Y_{n}(m)=(\tilde{X} \backslash v \widetilde{\Sigma}) \cup \varphi\left(Y_{n}(m) \backslash v \Sigma_{n}\right)
$$

along $\widetilde{\Sigma}$ and $\Sigma_{n}$ is simply connected, and satisfies

$$
\begin{aligned}
e\left(P_{n}^{m}(X)\right) & =e(X)+d+(8 a+2) t+8 b-8, \\
\sigma\left(P_{n}^{m}(X)\right) & =\sigma(X)-2 t-d, \\
\chi_{h}\left(P_{n}^{m}(X)\right) & =\chi_{h}(X)+2 a t+2 b-2, \\
c_{1}^{2}\left(P_{n}^{m}(X)\right) & =c_{1}^{2}(X)-d+(16 a-2) t+16 b-16, \\
b_{2}^{+}\left(P_{n}^{m}(X)\right) & =b_{2}^{+}(X)-b_{1}(X)+4 a t+4 b-4 \geq 3, \\
b_{2}^{-}\left(P_{n}^{m}(X)\right) & =b_{2}^{-}(X)-b_{1}(X)+d+(4 a+2) t+4 b-4 .
\end{aligned}
$$

If $\sigma\left(P_{n}^{m}(X)\right)$ is not divisible by 16 or if $2 t+d>0$, then $P_{n}^{m}(X)$ is nonspin and the set $\left\{P_{n}^{m}(X) \mid m \geq 1\right\}$ contains infinitely many homeomorphic but pairwise nondiffeomorphic irreducible 4-manifolds. When $m=1, P_{n}^{1}(X)$ is symplectic and irreducible. If $n=t a+b \geq 3$, then $P_{n}^{1}(X)$ contains disjoint symplectic tori $T_{1}$ and $T_{2}$ of self-intersection 0 satisfying $\pi_{1}\left(P_{n}^{1}(X) \backslash\left(T_{1} \cup T_{2}\right)\right)=1$. 
Proof. Recall from [Akhmedov and Park 2010a] that $e\left(Y_{n}(m)\right)=4 n-4$ and $\sigma\left(Y_{n}(m)\right)=0$ since torus surgeries change neither $e$ nor $\sigma$. Hence we have

$$
\begin{aligned}
e\left(P_{n}^{m}(X)\right) & =e(\tilde{X})+e\left(Y_{n}(m)\right)-2 e\left(\Sigma_{n}\right) \\
& =e(X)+2 t+d+4 n-4-2(2-2 n) \\
& =e(X)+2 t+d+8 n-8 \\
& =e(X)+2 t+d+8 t a+8 b-8, \\
\sigma\left(P_{n}^{m}(X)\right) & =\sigma(\tilde{X})+\sigma\left(Y_{n}(m)\right)=\sigma(X)-2 t-d .
\end{aligned}
$$

The other characteristic numbers can be computed from the formulas $\chi_{h}=\frac{1}{4}(e+\sigma)$, $c_{1}^{2}=2 e+3 \sigma, b_{2}^{+}=b_{1}-1+\frac{1}{2}(e+\sigma)$, and $b_{2}^{-}=b_{1}-1+\frac{1}{2}(e-\sigma)$.

To compute $\pi_{1}\left(P_{n}^{m}(X)\right)$, we first choose a standard presentation

$$
\pi_{1}\left(\Sigma_{n}\right)=\left\langle c_{1}, d_{1}, \ldots, c_{n}, d_{n} \mid \prod_{j=1}^{n}\left[c_{j}, d_{j}\right]=1\right\rangle .
$$

From the presentation of $\pi_{1}\left(Y_{n}(m)\right)$ in [Akhmedov and Park 2010a], we know that $\pi_{1}\left(Y_{n}(m)\right) /\langle z\rangle=1$, where $\langle z\rangle$ is the normal subgroup generated by the image $z$ of any one of the four generators $c_{1}, d_{1}, c_{2}, d_{2}$ of $\pi_{1}\left(\Sigma_{n}\right)$ under the inclusion induced homomorphism $\pi_{1}\left(\Sigma_{n}\right) \rightarrow \pi_{1}\left(Y_{n}(m)\right)$. We also know that any meridian of $\Sigma_{n}$ is conjugate to the image of $\left[a_{1}, b_{1}\right]\left[a_{2}, b_{2}\right]$ in $\pi_{1}\left(Y_{n}(m) \backslash v \Sigma_{n}\right)$, where $a_{i}$, $b_{i}(i=1,2)$ are the images of standard generators of $\pi_{1}\left(\Sigma_{2} \times\{\mathrm{pt}\}\right)$. All relations of $\pi_{1}\left(Y_{n}(m)\right)$ listed in [Akhmedov and Park 2010a], except $\left[a_{1}, b_{1}\right]\left[a_{2}, b_{2}\right]=1$, continue to hold in $\pi_{1}\left(Y_{n}(m) \backslash v \Sigma_{n}\right)$ since these relations come from torus surgeries that occur away from $v \Sigma_{n}$. Since $z=1$ still implies $a_{i}=b_{i}=1(i=1,2)$ in $\pi_{1}\left(Y_{n}(m) \backslash v \Sigma_{n}\right)$, we deduce that $\pi_{1}\left(Y_{n}(m) \backslash v \Sigma_{n}\right) /\langle z\rangle=1$.

When forming the generalized fiber sum $P_{n}^{m}(X)$, we choose the gluing diffeomorphism $\varphi$ such that the induced homomorphism $\varphi_{*}$ maps the element of $\pi_{1}\left(\widetilde{\Sigma}^{\|}\right)$ represented by a nonseparating vanishing cycle of the Lefschetz fibration $X$ to $z$, viewed as an element of $\pi_{1}\left(\Sigma_{n}^{\|}\right)$. Thus $z=1$ in $\pi_{1}\left(P_{n}^{m}(X)\right)$, which then implies that the inclusion induced homomorphism

$$
\pi_{1}\left(Y_{n}(m) \backslash v \Sigma_{n}\right) \longrightarrow \pi_{1}\left(P_{n}^{m}(X)\right)
$$

is trivial. Note that the inclusion induced homomorphism $\pi_{1}\left(\widetilde{\Sigma}^{\|}\right) \rightarrow \pi_{1}\left(P_{n}^{m}(X)\right)$ is also trivial since it can be factored through homomorphism (10) after $\widetilde{\Sigma}^{\|}$is identified with $\Sigma_{n}^{\|}$via $\varphi$. It follows from Lemma 7 that the inclusion induced homomorphism $\pi_{1}(\widetilde{X} \backslash v \widetilde{\Sigma}) \rightarrow \pi_{1}\left(P_{n}^{m}(X)\right)$ is trivial as well. By the Seifert - van Kampen theorem, we conclude that $\pi_{1}\left(P_{n}^{m}(X)\right)=1$.

If $2 t+d>0$, then $P_{n}^{m}(X)$ contains a genus 2 surface of self-intersection -1 that is the internal sum of a punctured exceptional sphere in $\widetilde{X} \backslash v \widetilde{\Sigma}$ and a punctured 
$\Sigma_{2}$ in $Y_{n}(m) \backslash v \Sigma_{n}$. In this case, the intersection form of $P_{n}^{m}(X)$ is odd and $P_{n}^{m}(X)$ is nonspin. Also recall that the signature of a spin 4-manifold is divisible by 16 according to Rohlin's theorem [1952].

Note that $e\left(P_{n}^{m}(X)\right)$ and $\sigma\left(P_{n}^{m}(X)\right)$ are independent of $m$. If $\sigma\left(P_{n}^{m}(X)\right)$ is not divisible by 16 or if $2 t+d>0$, then for fixed $n$, the set $\left\{P_{n}^{m}(X) \mid m \geq 1\right\}$ consists of homeomorphic simply connected nonspin 4-manifolds by Freedman's classification theorem (cf. [Freedman 1982]).

Since $Y_{n}(1)$ is symplectic, the corresponding fiber sum $P_{n}^{1}(\underset{\widetilde{X}}{(\widetilde{\Sigma})}$ is symplectic as well (cf. [Gompf 1995; McCarthy and Wolfson 1994]). Since $(\widetilde{X}, \widetilde{\Sigma})$ is a relatively minimal pair (i.e., every sphere of self-intersection -1 intersects $\widetilde{\Sigma}$ ) by Corollary 3 in [Li 1999], $P_{n}^{1}(X)$ is minimal by Usher's theorem [2006]. Recall from [Hamilton and Kotschick 2006; Kotschick 1997] that a simply connected minimal symplectic 4-manifold is irreducible, and thus $P_{n}^{1}(X)$ is irreducible.

Any Lefschetz fibration $X$ with fiber genus $a$ and base genus $b$ satisfies $b_{1}(X) \leq$ $2 a+2 b$. Since $X$ has at least one nonseparating vanishing cycle, we have $b_{1}(X)<$ $2 a+2 b \leq 2 a t+2 b$. Thus we deduce that $b_{2}^{+}\left(P_{n}^{m}(X)\right)>b_{2}^{+}(X) \geq 1$. Since $P_{n}^{1}(X)$ is symplectic and simply connected, $b_{2}^{+}\left(P_{n}^{1}(X)\right)=b_{2}^{+}\left(P_{n}^{m}(X)\right)$ is odd. It follows that $b_{2}^{+}\left(P_{n}^{m}(X)\right) \geq 3$ and the Seiberg-Witten invariant of $P_{n}^{m}(X)$ is well defined.

Let $Y_{0}$ denote the symplectic 4-manifold that is obtained by performing the same torus surgeries on $\Sigma_{2} \times \Sigma_{n}$ as for $Y_{n}(m)$, except $\left(a_{1}^{\prime \prime} \times d_{2}^{\prime}, d_{2}^{\prime},+m\right)$ surgery (cf. [Akhmedov and Park 2010a]). Let $P_{0}=\widetilde{X} \# \varphi Y_{0}$ be the generalized fiber sum of $\tilde{X}$ and $Y_{0}$ along $\widetilde{\Sigma}$ and $\Sigma_{n}$ using the same gluing diffeomorphism $\varphi$ that was used in the construction of $P_{n}^{m}(X)$. Note that $P_{0}$ is symplectic and minimal for the same reasons as $P_{n}^{1}(X)$. We have $b_{2}\left(P_{0}\right)=b_{2}\left(P_{n}^{m}(X)\right)+2$, and there is an orthogonal decomposition $H^{2}\left(P_{0} ; \mathbb{Z}\right)=H \oplus H^{\perp}$, where $H$ is the 2-dimensional hyperbolic summand generated by the Poincaré duals of $\left[a_{1} \times d_{2}\right]$ and $\left[b_{1} \times c_{2}\right]$. Using the adjunction inequality, we can easily see that every Seiberg-Witten basic class of $P_{0}$ lies in $H^{\perp}$.

Since $P_{n}^{m}(X)$ can be obtained from $P_{n}^{1}(X)$ by performing a $1 /(m-1)$ surgery on a null-homologous torus, we can apply the product formula in [Morgan et al. 1997] as in [Akhmedov et al. 2008; Fintushel et al. 2007; Szabó 1998] and deduce that there exist surjective homomorphisms

$$
\xi_{m}: H^{\perp} \longrightarrow H^{2}\left(P_{n}^{m}(X) ; \mathbb{Z}\right)
$$

that preserve the cup product pairing and satisfy

$$
\mathrm{SW}_{P_{n}^{m}(X)}\left(\xi_{m}\left(L_{0}\right)\right)=\mathrm{SW}_{P_{n}^{1}(X)}\left(\xi_{1}\left(L_{0}\right)\right)+(m-1) \mathrm{SW}_{P_{0}}\left(L_{0}\right),
$$

for every characteristic element $L_{0} \in H^{\perp} \subset H^{2}\left(P_{0} ; \mathbb{Z}\right)$. We note that the right side of (11) contains only one $\mathrm{SW}_{P_{0}}$ term for the reasons given in the proof of Corollary 2 in [Fintushel et al. 2007]. By a theorem of Taubes [1994], we have 
$\mathrm{SW}_{P_{0}}\left(c_{1}\left(P_{0}\right)\right)= \pm 1$. By setting $L_{0}=c_{1}\left(P_{0}\right)$ in (11) and observing that there are infinitely many values for the Seiberg-Witten invariants of $P_{n}^{m}(X)$, we conclude that $\left\{P_{n}^{m}(X) \mid m \geq 1\right\}$ contains infinitely many pairwise nondiffeomorphic 4-manifolds.

Next we prove that $P_{n}^{m}(X)$ is irreducible for all $m$ large enough, or more specifically when $\mathrm{SW}_{P_{n}^{m}(X)}\left(\xi_{m}\left(c_{1}\left(P_{0}\right)\right)\right) \neq 0$. We will argue the same way as in the proof of Theorem 5.4 in [Kotschick 1997]. Suppose $P_{n}^{m}(X)=M \# N$ is a connected sum of two smooth 4-manifolds $M$ and $N$. Both $M$ and $N$ are simply connected since $P_{n}^{m}(X)$ is. If $b_{2}^{+}(M)$ and $b_{2}^{+}(N)$ are both positive, then the Seiberg-Witten invariant of $P_{n}^{m}(X)$ is trivial (cf. [Witten 1994]), a contradiction. Without loss of generality, assume $b_{2}^{+}(N)=0$. If $b_{2}(N)=0$, then the simply connected 4-manifold $N$ must be homeomorphic to $S^{4}$ by Freedman's theorem in [Freedman 1982]. Thus it remains to rule out the case when $b_{2}(N)=b_{2}^{-}(N)>0$. In this case, the intersection form of $N$ is a nontrivial negative definite form, so by Donaldson's theorem in [Donaldson 1983], it is equivalent to the standard diagonal form. Let $e_{1}, \ldots, e_{b_{2}(N)}$ be a basis for $H^{2}(N ; \mathbb{Z})$ such that $e_{i}^{2}=-1$ for each $i=1, \ldots, b_{2}(N)$, and $e_{i} \cdot e_{j}=0$ when $i \neq j$. Using the neck pinching argument as in [Donaldson 1996; Kotschick 1997], we deduce that $M$ has nontrivial Seiberg-Witten invariant. Moreover, if $L$ is any Seiberg-Witten basic class of $M$, then the cohomology classes

$$
L+\sum_{i=1}^{b_{2}(N)} a_{i} e_{i}
$$

where $a_{i}= \pm 1$ for each $i=1, \ldots, b_{2}(N)$, are all Seiberg-Witten basic classes of $P_{n}^{m}(X)=M \# N$. Furthermore, every Seiberg-Witten basic class of $P_{n}^{m}(X)$ can be written as (12).

Let $L_{m}=\xi_{m}\left(c_{1}\left(P_{0}\right)\right)$ be a Seiberg-Witten basic class of $P_{n}^{m}(X)$. By changing any basis element $e_{i}$ to $-e_{i}$ if necessary, we can assume that $L_{m}=L-e_{1}-\cdots-e_{b_{2}(N)}$ for some $L$. Thus $L_{m}+2 e_{1}=L+e_{1}-e_{2}-\cdots-e_{b_{2}(N)}$ is also a Seiberg-Witten basic class of $P_{n}^{m}(X)$. By the adjunction inequality, we can assume that $\xi_{1}\left(c_{1}\left(P_{0}\right)\right)=$ $c_{1}\left(P_{n}^{1}(X)\right)$. It now follows from (11) that there exists $\bar{e}_{1} \in \xi_{m}^{-1}\left(e_{1}\right) \subset H^{\perp}$ such that $c_{1}\left(P_{n}^{1}(X)\right)+2 \xi_{1}\left(\bar{e}_{1}\right)$ or $c_{1}\left(P_{0}\right)+2 \bar{e}_{1}$ is a Seiberg-Witten basic class of $P_{n}^{1}(X)$ or $P_{0}$, respectively. By a theorem of Taubes [1996], we can then deduce that the Poincaré dual of $\xi_{1}\left(\bar{e}_{1}\right)$ or $\bar{e}_{1}$ is represented by an embedded symplectic sphere of self-intersection -1 in $P_{n}^{1}(X)$ or $P_{0}$, respectively (cf. Remark 10.1.16(b) in [Gompf and Stipsicz 1999]). This implies that $P_{n}^{1}(X)$ or $P_{0}$ is not minimal, a contradiction.

Finally, if $n \geq 3$, then $Y_{n}(1)$ contains $2 n-4$ pairs of geometrically dual Lagrangian tori that are all disjoint from $\Sigma_{n}$. The images of these $4 n-8$ tori in the fiber sum $P_{n}^{1}(X)$ are again Lagrangian submanifolds (cf. [Gompf 1995]). Let $T_{1}$ and $T_{2}$ be two of these $4 n-8$ Lagrangian tori in $P_{n}^{1}(X)$ that are not geometrically dual to each other. By perturbing the symplectic form on $P_{n}^{1}(X)$, we can turn both $T_{1}$ and $T_{2}$ into symplectic submanifolds of $P_{n}^{1}(X)$ (cf. [Gompf 1995, Lemma 1.6]). 
To show $\pi_{1}\left(P_{n}^{1}(X) \backslash\left(T_{1} \cup T_{2}\right)\right)=1$, it will be convenient to fix $T_{1}$ and $T_{2}$, say $T_{1}=a_{1}^{\prime} \times c_{3}^{\prime \prime}$ and $T_{2}=a_{2}^{\prime} \times d_{3}^{\prime \prime}$. Here, $a_{1}^{\prime}, a_{2}^{\prime}, c_{3}^{\prime \prime}$ and $d_{3}^{\prime \prime}$ are parallel copies of $a_{1}$, $a_{2}, c_{3}$ and $d_{3}$ as defined in [Fintushel et al. 2007]. Then $\pi_{1}\left(P_{n}^{1}(X) \backslash\left(T_{1} \cup T_{2}\right)\right)$ is normally generated by meridians of $T_{1}$ and $T_{2}$, which are all conjugate to the commutators $\left[b_{1}^{-1}, d_{3}\right]$ or $\left[b_{2}^{-1}, c_{3}\right]$. Note that the generators $b_{1}, b_{2}, c_{3}$ and $d_{3}$ are still trivial in $\pi_{1}\left(P_{n}^{1}(X) \backslash\left(T_{1} \cup T_{2}\right)\right)$ since the Luttinger surgery relations in Section 2 of [Akhmedov and Park 2010a] still hold true in $\pi_{1}\left(P_{n}^{1}(X) \backslash\left(T_{1} \cup T_{2}\right)\right)$. It follows that meridians of $T_{1}$ and $T_{2}$ are all trivial and hence $\pi_{1}\left(P_{n}^{1}(X) \backslash\left(T_{1} \cup T_{2}\right)\right)=$ $\pi_{1}\left(P_{n}^{1}(X)\right)=1$.

Instead of using $Y_{n}(m)$ summand in generalized fiber sum (9), we may use $Y_{n-2}(m) \# 2 \overline{\mathbb{C P}}^{2}$ when $n \geq 4$. Specifically, we resolve the intersection between $\Sigma_{2}$ and $\Sigma_{n-2}$ in $Y_{n-2}(m)$ to obtain a genus $n$ submanifold of $Y_{n-2}(m)$ with selfintersection 2. Next we blow up two points on this submanifold to obtain a genus $n$ submanifold $\Sigma_{n}^{\prime}$ of self-intersection 0 in $Y_{n-2}(m) \# 2 \overline{\mathbb{C P}}^{2}$. When $m=1$, the resolution and the blowups can be performed symplectically, and hence $\left(Y_{n-2}(1) \#\right.$ $\left.2 \overline{\mathbb{C P}}^{2}, \Sigma_{n}^{\prime}\right)$ is a relatively minimal pair of symplectic manifolds. The advantage of using $Y_{n-2}(m) \# 2 \overline{\mathbb{C P}}^{2}$ summand is that the resulting generalized fiber sum has slightly smaller characteristic numbers than $P_{n}^{m}(X)$.

Theorem 9. Let $f: X \rightarrow \Sigma_{b}$ be a relatively minimal Lefschetz fibration as above having at least one nonseparating vanishing cycle. Suppose that $n=t a+b \geq 4$. For a suitable choice of the gluing diffeomorphism $\psi: \partial(v \widetilde{\Sigma}) \rightarrow \partial\left(v \Sigma_{n}^{\prime}\right)$, the generalized fiber sum

$$
\begin{aligned}
Q_{n}^{m}(X) & =\widetilde{X} \#_{\psi}\left(Y_{n-2}(m) \# 2 \overline{\mathbb{C P}}^{2}\right) \\
& =(\tilde{X} \backslash v \widetilde{\Sigma}) \cup_{\psi}\left(\left(Y_{n-2}(m) \# 2 \overline{\mathbb{C P}}^{2}\right) \backslash v \Sigma_{n}^{\prime}\right)
\end{aligned}
$$

along $\widetilde{\Sigma}$ and $\Sigma_{n}^{\prime}$ is simply connected, nonspin, and satisfies

$$
\begin{aligned}
e\left(Q_{n}^{m}(X)\right) & =e(X)+d+(8 a+2) t+8 b-14, \\
\sigma\left(Q_{n}^{m}(X)\right) & =\sigma(X)-2 t-d-2, \\
\chi_{h}\left(Q_{n}^{m}(X)\right) & =\chi_{h}(X)+2 a t+2 b-4, \\
c_{1}^{2}\left(Q_{n}^{m}(X)\right) & =c_{1}^{2}(X)-d+(16 a-2) t+16 b-34, \\
b_{2}^{+}\left(Q_{n}^{m}(X)\right) & =b_{2}^{+}(X)-b_{1}(X)+4 a t+4 b-8 \geq 3, \\
b_{2}^{-}\left(Q_{n}^{m}(X)\right) & =b_{2}^{-}(X)-b_{1}(X)+d+(4 a+2) t+4 b-6 .
\end{aligned}
$$

The set $\left\{Q_{n}^{m}(X) \mid m \geq 1\right\}$ contains infinitely many homeomorphic but pairwise nondiffeomorphic irreducible 4-manifolds. When $m=1, Q_{n}^{1}(X)$ is symplectic and irreducible. If $n=t a+b \geq 5$, then $Q_{n}^{1}(X)$ contains disjoint symplectic tori $T_{1}^{\prime}$ and $T_{2}^{\prime}$ of self-intersection 0 satisfying $\pi_{1}\left(Q_{n}^{1}(X) \backslash\left(T_{1}^{\prime} \cup T_{2}^{\prime}\right)\right)=1$. 
Proof. We compute that

$$
\begin{aligned}
e\left(Q_{n}^{m}(X)\right) & =e(\tilde{X})+e\left(Y_{n-2}(m) \# 2 \overline{\mathbb{C P}}^{2}\right)-2 e\left(\Sigma_{n}^{\prime}\right) \\
& =e(X)+2 t+d+4(n-2)-4+2-2(2-2 n) \\
& =e(X)+2 t+d+8 n-14 \\
& =e(X)+2 t+d+8 t a+8 b-14 \\
\sigma\left(Q_{n}^{m}(X)\right) & =\sigma(\tilde{X})+\sigma\left(Y_{n-2}(m) \# 2 \overline{\mathbb{C P}}^{2}\right)=\sigma(X)-2 t-d-2 .
\end{aligned}
$$

The other characteristic numbers can be computed from these as before.

Since the exceptional sphere of a blowup intersects $\Sigma_{n}^{\prime}$ once transversely, any meridian of $\Sigma_{n}^{\prime}$ is null-homotopic in the complement of a tubular neighborhood $\nu \Sigma_{n}^{\prime}$. Hence we conclude that

$$
\pi_{1}\left(\left(Y_{n-2}(m) \# 2 \overline{\mathbb{C P}}^{2}\right) \backslash v \Sigma_{n}^{\prime}\right)=\pi_{1}\left(Y_{n-2}(m) \# 2 \overline{\mathbb{C P}}^{2}\right)=\pi_{1}\left(Y_{n-2}(m)\right) .
$$

From [Akhmedov and Park 2010a], we know that $\pi_{1}\left(Y_{n-2}(m)\right) /\langle z\rangle=1$, where $z$ is the image of any one of the generators $c_{1}, d_{1}, c_{2}, d_{2}$ of $\pi_{1}\left(\Sigma_{n-2}\right)$ under the inclusion induced homomorphism.

Let $\widetilde{\Sigma}^{\|}$and $\Sigma_{n}^{\prime \prime}$ denote parallel copies of $\widetilde{\Sigma}$ and $\Sigma_{n}^{\prime}$ in the boundaries $\partial(v \widetilde{\Sigma})$ and $\partial\left(v \Sigma_{n}^{\prime}\right)$, respectively. When forming the generalized fiber sum $Q_{n}^{m}(X)$, we choose the gluing diffeomorphism $\psi$ such that $\psi_{*}$ maps the element of $\pi_{1}\left(\widetilde{\Sigma}^{\|}\right)$ represented by a nonseparating vanishing cycle of $X$ to $z$, viewed as an element of $\pi_{1}\left(\Sigma_{n}^{\prime \prime \prime}\right)$. Thus $z=1$ in $\pi_{1}\left(Q_{n}^{m}(X)\right)$, which then implies that the inclusion induced homomorphism

$$
\pi_{1}\left(\left(Y_{n-2}(m) \# 2 \overline{\mathbb{C P}}^{2}\right) \backslash v \Sigma_{n}^{\prime}\right) \longrightarrow \pi_{1}\left(Q_{n}^{m}(X)\right)
$$

is trivial. Note that the inclusion induced homomorphism $\pi_{1}\left(\widetilde{\Sigma}^{\|}\right) \rightarrow \pi_{1}\left(Q_{n}^{m}(X)\right)$ is also trivial since it can be factored through homomorphism (13) after $\widetilde{\Sigma}^{\|}$is identified with $\Sigma_{n}^{\prime \prime}$. It follows from Lemma 7 that the inclusion induced homomorphism $\pi_{1}(\tilde{X} \backslash v \widetilde{\Sigma}) \rightarrow \pi_{1}\left(Q_{n}^{m}(X)\right)$ is trivial as well. By Seifert-van Kampen theorem, we conclude that $\pi_{1}\left(Q_{n}^{m}(X)\right)=1$.

$Q_{n}^{m}(X)$ is nonspin since it contains a surface of self-intersection -1 and genus $a>0$, namely the internal sum of the image of a punctured fiber of $X$ in $\widetilde{X} \backslash v \widetilde{\Sigma}$ and a punctured exceptional sphere in $\left(Y_{n-2}(m) \# 2 \overline{\mathbb{C P}}^{2}\right) \backslash v \Sigma_{n}^{\prime}$. Since $Y_{n-2}(1) \# 2 \overline{\mathbb{C P}}^{2}$ is symplectic, the corresponding fiber sum $Q_{n}^{1}(X)$ is symplectic as well. The irreducibility of $Q_{n}^{1}(X)$ and the fact that $\left\{Q_{n}^{m}(X) \mid m \geq 1\right\}$ contains infinitely many homeomorphic but pairwise nondiffeomorphic irreducible 4-manifolds can be proved exactly the same way as in the proof of Theorem 8 .

Finally, if $n \geq 5$, then $Y_{n-2}$ (1) contains $2 n-8$ pairs of geometrically dual Lagrangian tori. The images of these $4 n-16$ tori in the blowup $Y_{n-2}(1) \# 2 \overline{\mathbb{C P}}^{2}$ are 
disjoint from $\Sigma_{n}^{\prime}$, and hence their images in $Q_{n}^{1}(X)$ are Lagrangian submanifolds of $Q_{n}^{1}(X)$. Let $T_{1}^{\prime}$ and $T_{2}^{\prime}$ denote two of these $4 n-16$ Lagrangian tori, say $T_{1}^{\prime}=a_{1}^{\prime} \times c_{3}^{\prime \prime}$ and $T_{2}^{\prime}=a_{2}^{\prime} \times d_{3}^{\prime \prime}$. By perturbing the symplectic form on $Q_{n}^{1}(X)$, we can turn both $T_{1}^{\prime}$ and $T_{2}^{\prime}$ into symplectic submanifolds of $Q_{n}^{1}(X)$. We can deduce that $\pi_{1}\left(Q_{n}^{1}(X) \backslash\left(T_{1}^{\prime} \cup T_{2}^{\prime}\right)\right)=1$ in exactly the same way as in the proof of Theorem 8 .

For comparison, we note that

$$
\begin{aligned}
& e\left(Q_{n}^{m}(X)\right)=e\left(P_{n}^{m}(X)\right)-6, \quad \sigma\left(Q_{n}^{m}(X)\right)=\sigma\left(P_{n}^{m}(X)\right)-2, \\
& \chi_{h}\left(Q_{n}^{m}(X)\right)=\chi_{h}\left(P_{n}^{m}(X)\right)-2, \quad c_{1}^{2}\left(Q_{n}^{m}(X)\right)=c_{1}^{2}\left(P_{n}^{m}(X)\right)-18, \\
& b_{2}^{+}\left(Q_{n}^{m}(X)\right)=b_{2}^{+}\left(P_{n}^{m}(X)\right)-4, \quad b_{2}^{-}\left(Q_{n}^{m}(X)\right)=b_{2}^{-}\left(P_{n}^{m}(X)\right)-2 \text {. }
\end{aligned}
$$

Remark 10. The irreducible symplectic 4-manifolds $M$ and $N$ (homeomorphic to $47 \mathbb{C P} \mathbb{P}^{2} \# 45 \overline{\mathbb{C P}}^{2}$ and $51 \mathbb{C P}^{2} \# 47 \overline{\mathbb{C P}}^{2}$, respectively) in Section 4 of [Akhmedov and Park 2008] are respectively equal to $Q_{n}^{1}(X)$ and $P_{n}^{1}(X)$ with $a=7, b=2, t=1$, $d=-2, n=9, e(X)=36$, and $\sigma(X)=4$.

\section{Simply connected 4-manifolds with positive signature}

We now apply Theorems 8 and 9 to Lefschetz fibrations in Sections 2 and 3 to obtain new families of simply connected irreducible 4-manifolds with positive signature.

Example 11. For each triple of positive integers $g, u_{1}, u_{2}$, recall from Example 3 that there is a Lefschetz fibration $f_{u_{1}, u_{2}}: X_{u_{1}, u_{2}}^{g} \rightarrow \Sigma_{b}$ such that the genus of a regular fiber is $a=1+\frac{1}{2} u_{2}(g+1)(3 g-2)$ and there is a section whose image is a surface of genus $b=u_{1}(g-1)+1$ and self-intersection $d=-2 u_{1}$. Since $2 t+d \geq 0$, we require $t \geq u_{1}$. Let

$$
n=t+\frac{1}{2} t u_{2}(g+1)(3 g-2)+u_{1}(g-1)+1 .
$$

Applying Theorem 8 to $f_{u_{1}, u_{2}}: X_{u_{1}, u_{2}}^{g} \rightarrow \Sigma_{b}$, we obtain a family of simply connected 4-manifolds $P_{n}^{m}\left(X_{u_{1}, u_{2}}^{g}\right)$, with $m \geq 1$ and $n \geq 3$, satisfying

$$
\begin{gathered}
e\left(P_{n}^{m}\left(X_{u_{1}, u_{2}}^{g}\right)\right)=2 u_{1} u_{2}(g+1)\left(3 g^{2}-5 g+4\right) \\
+4 t u_{2}(g+1)(3 g-2)+8 u_{1} g+10 t-10 u_{1}, \\
\sigma\left(P_{n}^{m}\left(X_{u_{1}, u_{2}}^{g}\right)\right)=\frac{2}{3} u_{1} u_{2}(g+1)\left(g^{2}+2 g-6\right)-2 t+2 u_{1}, \\
\chi_{h}\left(P_{n}^{m}\left(X_{u_{1}, u_{2}}^{g}\right)\right)=\frac{1}{6} u_{1} u_{2}(g+1)\left(10 g^{2}-13 g+6\right) \\
+t u_{2}(g+1)(3 g-2)+2 t+2 u_{1}(g-1), \\
c_{1}^{2}\left(P_{n}^{m}\left(X_{u_{1}, u_{2}}^{g}\right)\right)=2 u_{1} u_{2}(g+1)\left(7 g^{2}-8 g+2\right) \\
+8 t u_{2}(g+1)(3 g-2)+16 u_{1} g+14 t-14 u_{1},
\end{gathered}
$$




$$
\begin{aligned}
b_{2}^{+}\left(P_{n}^{m}\left(X_{u_{1}, u_{2}}^{g}\right)\right)=\frac{1}{3} u_{1} u_{2}(g+1) & \left(10 g^{2}-13 g+6\right) \\
+ & 2 t u_{2}(g+1)(3 g-2)+4 t+4 u_{1}(g-1)-1,
\end{aligned}
$$

$$
\begin{aligned}
b_{2}^{-}\left(P_{n}^{m}\left(X_{u_{1}, u_{2}}^{g}\right)\right)=\frac{1}{3} u_{1} u_{2}(g+1)\left(8 g^{2}-17 g+18\right) \\
+2 t u_{2}(g+1)(3 g-2)+4 u_{1} g+6 t-6 u_{1}-1 .
\end{aligned}
$$

From Theorem 9, we obtain another family of simply connected nonspin 4-manifolds $Q_{n}^{m}\left(X_{u_{1}, u_{2}}^{g}\right)$, with $m \geq 1$ and $n \geq 5$, whose characteristic numbers can be computed from (14) (15), and (16). Moreover, when $m=1$, both $P_{n}^{1}\left(X_{u_{1}, u_{2}}^{g}\right)$ and $Q_{n}^{1}\left(X_{u_{1}, u_{2}}^{g}\right)$ are irreducible symplectic 4-manifolds and contain symplectic tori $T_{j}$ and $T_{j}^{\prime}(j=$ $1,2)$ of self-intersection 0 such that

$$
\pi_{1}\left(P_{n}^{1}\left(X_{u_{1}, u_{2}}^{g}\right) \backslash\left(T_{1} \cup T_{2}\right)\right)=1 \quad \text { and } \quad \pi_{1}\left(Q_{n}^{1}\left(X_{u_{1}, u_{2}}^{g}\right) \backslash\left(T_{1}^{\prime} \cup T_{2}^{\prime}\right)\right)=1 .
$$

Example 12. For each quadruple of positive integers $p, v, u_{1}, u_{2}$ with odd $p \geq 3$, recall from Example 5 that there is a Lefschetz fibration $f_{u_{1}, u_{2}}: W_{u_{1}, u_{2}}^{p, v} \rightarrow \Sigma_{b}$ such that the genus of a regular fiber is $a=1+\frac{1}{2} p u_{2}[(v+1) p-v-3]$ and there is a section whose image is a surface of genus $b=1+u_{1}[-1+v(p-1) / 2]$ and self-intersection $d=-u_{1} v$. Since $2 t+d \geq 0$, we require

$$
t \geq\left\lceil u_{1} v / 2\right\rceil,
$$

where $\lceil x\rceil=\min \{k \in \mathbb{Z} \mid k \geq x\}$. From Theorems 8 and 9, we obtain two families of simply connected 4-manifolds $P_{n}^{m}\left(W_{u_{1}, u_{2}}^{p, v}\right)$ and $Q_{n}^{m}\left(W_{u_{1}, u_{2}}^{p, v}\right)$ with $m \geq 1$ and

$$
n=t+\frac{1}{2} t p u_{2}[(v+1) p-v-3]+u_{1}[-1+v(p-1) / 2]+1 \geq 5 .
$$

We compute that

$$
\begin{gathered}
e\left(P_{n}^{m}\left(W_{u_{1}, u_{2}}^{p, v}\right)\right)=p u_{1} u_{2}\left[\left(v^{2}+v\right) p^{2}-2\left(v^{2}+3 v+1\right) p+v^{2}+6 v+8\right] \\
+4 t u_{2}(v+1) p^{2}+4\left[u_{1} v-t u_{2}(v+3)\right] p+10 t-5 u_{1} v-8 u_{1}, \\
\sigma\left(P_{n}^{m}\left(W_{u_{1}, u_{2}}^{p, v}\right)\right)=\frac{1}{3} p u_{1} u_{2}\left(v p^{2}-4 v-6\right)-2 t+u_{1} v, \\
\chi_{h}\left(P_{n}^{m}\left(W_{u_{1}, u_{2}}^{p, v}\right)\right)=\frac{1}{12} p u_{1} u_{2}\left[\left(3 v^{2}+4 v\right) p^{2}-6\left(v^{2}+3 v+1\right) p+3 v^{2}+14 v+18\right] \\
+t u_{2}(v+1) p^{2}+\left[u_{1} v-t u_{2}(v+3)\right] p+2 t-u_{1} v-2 u_{1}, \\
c_{1}^{2}\left(P_{n}^{m}\left(W_{u_{1}, u_{2}}^{p, v}\right)\right)=p u_{1} u_{2}\left[\left(2 v^{2}+3 v\right) p^{2}-4\left(v^{2}+3 v+1\right) p+2 v^{2}+8 v+10\right] \\
+8 t u_{2}(v+1) p^{2}+8\left[u_{1} v-t u_{2}(v+3)\right] p+14 t-7 u_{1} v-16 u_{1}, \\
b_{2}^{+}\left(P_{n}^{m}\left(W_{u_{1}, u_{2}}^{p, v}\right)\right)=\frac{1}{6} p u_{1} u_{2}\left[\left(3 v^{2}+4 v\right) p^{2}-6\left(v^{2}+3 v+1\right) p+3 v^{2}+14 v+18\right] \\
+2 t u_{2}(v+1) p^{2}+2\left[u_{1} v-t u_{2}(v+3)\right] p+4 t-2 u_{1} v-4 u_{1}-1, \\
b_{2}^{-}\left(P_{n}^{m}\left(W_{u_{1}, u_{2}}^{p, v}\right)\right)=\frac{1}{6} p u_{1} u_{2}\left[\left(3 v^{2}+2 v\right) p^{2}-6\left(v^{2}+3 v+1\right) p+3 v^{2}+22 v+30\right] \\
+2 t u_{2}(v+1) p^{2}+2\left[u_{1} v-t u_{2}(v+3)\right] p+6 t-3 u_{1} v-4 u_{1}-1 .
\end{gathered}
$$


The characteristic numbers of $Q_{n}^{m}\left(W_{u_{1}, u_{2}}^{p, v}\right)$ can be computed from these values via (14). When $m=1$, both $P_{n}^{1}\left(W_{u_{1}, u_{2}}^{p, v}\right)$ and $Q_{n}^{1}\left(W_{u_{1}, u_{2}}^{p, v}\right)$ are irreducible symplectic 4-manifolds and contain symplectic tori $T_{j}$ and $T_{j}^{\prime}(j=1,2)$ of self-intersection 0 such that $\pi_{1}\left(P_{n}^{1}\left(W_{u_{1}, u_{2}}^{p, v}\right) \backslash\left(T_{1} \cup T_{2}\right)\right)=1$ and $\pi_{1}\left(Q_{n}^{1}\left(W_{u_{1}, u_{2}}^{p, v}\right) \backslash\left(T_{1}^{\prime} \cup T_{2}^{\prime}\right)\right)=1$.

\section{Upper bounds for the lower bound}

We start this section by giving a more rigorous definition of $\lambda(\sigma)$ from the introduction.

Definition 13. Given an integer $\sigma \geq 0$, let $\lambda(\sigma)$ be the smallest positive integer with the following properties.

(i) $\lambda(\sigma) \geq\lceil(\sigma+1) / 2\rceil$.

(ii) Every point $\left(\chi_{h}, c_{1}^{2}\right)$ on the line $c_{1}^{2}=8 \chi_{h}+\sigma$ satisfying $\chi_{h} \geq \lambda(\sigma)$ is realized as $\left(\chi_{h}\left(M_{i}\right), c_{1}^{2}\left(M_{i}\right)\right)$, where $\left\{M_{i} \mid i \in \mathbb{Z}\right\}$ is an infinite family of homeomorphic but pairwise nondiffeomorphic closed simply connected nonspin irreducible 4-manifolds such that $M_{i}$ is symplectic for each $i \geq 0$ and $M_{i}$ is nonsymplectic for each $i<0$.

As in the introduction, we make the following conjecture.

Conjecture 14. $\lambda(\sigma)=\lceil(\sigma+1) / 2\rceil$ for every integer $\sigma \geq 0$.

Our goal in this section is to calculate explicit upper bounds on $\lambda(\sigma)$ for many small values of $\sigma$. First we restate a result from [Akhmedov and Park 2008] (see also [Akhmedov et al. 2010a, Theorem 23; Akhmedov and Park 2010a, Theorem 2]).

Theorem 15 [Akhmedov and Park 2008, Theorem 5.3]. Let X be a closed symplectic 4-manifold that contains a symplectic torus $T$ of self-intersection 0 . Let $\nu T$ be a tubular neighborhood of $T$ and $\partial(\nu T)$ its boundary. Suppose that the homomorphism $\pi_{1}(\partial(v T)) \rightarrow \pi_{1}(X \backslash v T)$ induced by the inclusion is trivial. Then for any pair of integers $(\chi, c)$ satisfying

$$
\chi \geq 1 \text { and } 0 \leq c \leq 8 \chi,
$$

there exists a symplectic 4-manifold $Y$ with $\pi_{1}(Y)=\pi_{1}(X)$,

$$
\chi_{h}(Y)=\chi_{h}(X)+\chi \text { and } c_{1}^{2}(Y)=c_{1}^{2}(X)+c .
$$

Moreover, if $X$ is minimal then $Y$ is minimal as well. If $c<8 \chi$, or if $c=8 \chi$ and $X$ has an odd intersection form, then the corresponding $Y$ has an odd indefinite intersection form.

The next theorem gives us a means for constructing infinitely many distinct smooth structures on some topological 4-manifolds. 
Theorem 16. Let $Y$ be a closed simply connected minimal symplectic 4-manifold with $b_{2}^{+}(Y)>1$. Assume that $Y$ contains a symplectic torus $T$ of self-intersection 0 such that $\pi_{1}(Y \backslash T)=1$. Then there exist an infinite family of pairwise nondiffeomorphic irreducible symplectic 4-manifolds and an infinite family of pairwise nondiffeomorphic irreducible nonsymplectic 4-manifolds, all of which are homeomorphic to $Y$.

Proof. We can perform a knot surgery on $Y$ along $T$ using a knot $K \subset S^{3}$ (see [Fintushel and Stern 2009, Lecture 3]). Let $Y_{K}$ denote the resulting 4-manifold. Since $\pi_{1}(Y \backslash T)=1, Y_{K}$ is homeomorphic to $Y$. By varying the knot $K$, we obtain infinitely many pairwise nondiffeomorphic 4-manifolds. If $K$ is a fibered knot, then $Y_{K}$ can be viewed as a symplectic fiber sum [Fintushel and Stern 1998], is minimal by Usher's theorem [2006], and hence is irreducible [Hamilton and Kotschick 2006; Kotschick 1997].

Given an integer $k \neq 0$, let $T(k)$ denote the $k$-twist knot on page 372 of [Fintushel and Stern 1998] with Alexander polynomial $k t-(2 k+1)+k t^{-1}$. If $k= \pm 1$, then $T( \pm 1)$ is fibered, and thus $Y_{T( \pm 1)}$ is symplectic and irreducible. If $k \neq 0, \pm 1$, then $Y_{T(k)}$ is nonsymplectic. It only remains to prove that $Y_{T(k)}$ is irreducible when $k \neq 0, \pm 1$. We will argue the same way as in the proof of Theorem 8 . The computation of the Seiberg-Witten invariant of $Y_{T(k)}$ in [Fintushel and Stern 2009] implies that there exists an isomorphism $\xi_{T(k)}: H^{2}\left(Y_{T(1)} ; \mathbb{Z}\right) \longrightarrow H^{2}\left(Y_{T(k)} ; \mathbb{Z}\right)$ that preserves the cup product pairing and restricts to a one-to-one correspondence between the Seiberg-Witten basic classes of $Y_{T(1)}$ and $Y_{T(k)}$. Suppose that $Y_{T(k)}$ is not irreducible. Then there will be some $e_{1} \in H^{2}\left(Y_{T(k)} ; \mathbb{Z}\right)$ such that $e_{1}^{2}=-1$ and $\xi_{T(k)}\left(c_{1}\left(Y_{T(1)}\right)\right)+2 e_{1}$ is a Seiberg-Witten basic class of $Y_{T(k)}$. This will imply that $c_{1}\left(Y_{T(1)}\right)+2 \xi_{T(k)}^{-1}\left(e_{1}\right)$ is a Seiberg-Witten basic class of $Y_{T(1)}$. By a result of Taubes [1996], we can then conclude that the Poincaré dual of $\xi_{T(k)}^{-1}\left(e_{1}\right)$ is represented by an embedded symplectic sphere of self-intersection -1 in $Y_{T(1)}$. Hence $Y_{T(1)}$ is not minimal, a contradiction.

By combining Theorems 15 and 16, we may deduce the following.

Corollary 17. Let $X$ be a closed simply connected nonspin minimal symplectic 4manifold with $b_{2}^{+}(X)>1$ and $\sigma(X) \geq 0$. Assume that $X$ contains disjoint symplectic tori $T_{1}$ and $T_{2}$ of self-intersection 0 such that $\pi_{1}\left(X \backslash\left(T_{1} \cup T_{2}\right)\right)=1$. Suppose $\sigma$ is a fixed integer satisfying $0 \leq \sigma \leq \sigma(X)$. If $\lceil x\rceil=\min \{k \in \mathbb{Z} \mid k \geq x\}$ and if we define

$$
\ell(\sigma)=\left\lceil\frac{\sigma(X)-\sigma}{8}-1\right\rceil
$$

then

$$
\lambda(\sigma) \leq \chi_{h}(X)+\ell(\sigma)+1 .
$$


In other words, if $k$ is any odd integer satisfying $k \geq b_{2}^{+}(X)+2 \ell(\sigma)+2$, then there exist an infinite family of pairwise nondiffeomorphic irreducible symplectic 4-manifolds and an infinite family of pairwise nondiffeomorphic irreducible nonsymplectic 4-manifolds, all of which are homeomorphic to $k \mathbb{C P} \mathbb{P}^{2} \#(k-\sigma) \overline{\mathbb{C P}}^{2}$. Proof. We can write $\sigma(X)-\sigma=8 \ell(\sigma)+r(\sigma)$ for integers $\ell(\sigma)$ and $r(\sigma)$ satisfying $\ell(\sigma) \geq-1$ and $1 \leq r(\sigma) \leq 8$. Since $\pi_{1}\left(X \backslash \nu T_{1}\right)=1$, we can apply Theorem 15 to the pair, $X$ and $T_{1}$. Let $(\chi, c)$ and $Y$ be as in the conclusion of Theorem 15. Since $\pi_{1}(Y)=\pi_{1}(X)=1$, we have $b_{2}^{+}(Y)=b_{2}^{+}(X)+2 \chi$ and $b_{2}^{-}(Y)=b_{2}^{-}(X)+10 \chi-c$. By Freedman's classification theorem [1982], $Y$ must be homeomorphic to

$$
\left(b_{2}^{+}(X)+2 \chi\right) \mathbb{C P}^{2} \#\left(b_{2}^{-}(X)+10 \chi-c\right) \overline{\mathbb{C P}}^{2} .
$$

By setting $c=8 \chi+\sigma-\sigma(X)$ in (17), we obtain a minimal symplectic 4-manifold $Y$ that is homeomorphic to $k \mathbb{C P}^{2} \#(k-\sigma) \overline{\mathbb{C P}}^{2}$, where $k=b_{2}^{+}(X)+2 \chi$. Since $c$ is nonnegative, we must have $8 \chi+\sigma-\sigma(X)=8(\chi-\ell(\sigma))-r(\sigma) \geq 0$, which implies that $\chi \geq \ell(\sigma)+1$. It follows that $\chi_{h}(Y) \geq \chi_{h}(X)+\ell(\sigma)+1$ and $k \geq$ $b_{2}^{+}(X)+2 \ell(\sigma)+2$.

We recall from [Akhmedov et al. 2010a; Akhmedov and Park 2008; 2010a] that for each pair of integers $(\chi, c)$ satisfying (17), there exist a minimal symplectic 4-manifold $Z$ with $\chi_{h}(Z)=\chi, c_{1}^{2}(Z)=c$, and a symplectic torus $T^{\prime \prime} \subset Z$ of self-intersection 0 such that $Y$ is the generalized fiber sum of $X$ and $Z$ along $T_{1}$ and $T^{\prime \prime}$. Note that $T_{2} \subset\left(X \backslash v T_{1}\right) \subset Y$ is a symplectic torus of self-intersection 0 in $Y$ (cf. [Gompf and Stipsicz 1999, Theorem 10.2.1]). Since $\pi_{1}\left(X \backslash\left(v T_{1} \cup T_{2}\right)\right)=1$, we have $\pi_{1}\left(Y \backslash T_{2}\right)=1$. We can now apply Theorem 16 to the pair, $Y$ and $T_{2}$, and conclude that there are infinitely many distinct smooth structures on $Y$.

Next we show that $\lambda(\sigma)$ is subadditive in the following sense.

Corollary 18. Let $\sigma_{1}$ and $\sigma_{2}$ be positive integers such that $\sigma_{1}+\sigma_{2}$ is not divisible by 16. For each $j=1,2$, suppose that there exists a closed simply connected nonspin minimal symplectic 4-manifold $N_{j}$ containing a symplectic torus $T_{j} \subset N_{j}$ of self-intersection 0 such that

(i) $\pi_{1}\left(N_{j} \backslash T_{j}\right)=1$,

(ii) $\chi_{h}\left(N_{j}\right)=\lambda\left(\sigma_{j}\right)$, and $\sigma\left(N_{j}\right)=\sigma_{j}$.

Then we have $\lambda\left(\sigma_{1}+\sigma_{2}\right) \leq \lambda\left(\sigma_{1}\right)+\lambda\left(\sigma_{2}\right)$.

Proof. Let $X$ be the generalized fiber sum of $N_{1}$ and $N_{2}$ along $T_{1}$ and $T_{2}$. It is easy to check that $X$ is a closed simply connected minimal symplectic 4-manifold. Since

$$
\sigma(X)=\sigma\left(N_{1}\right)+\sigma\left(N_{2}\right)=\sigma_{1}+\sigma_{2} \not \equiv 0(\bmod 16),
$$

$X$ is nonspin by Rohlin's theorem [1952]. Let $T$ be a parallel copy of $T_{1}$ (and $T_{2}$ ) in $X$. From (i), there are topological disks bounding the meridians of $T_{1}$ and $T_{2}$, 
and these disks can be glued together to form a topological sphere that intersects $T$ transversely once. It follows that $\pi_{1}(X \backslash T)=1$ and thus we can apply Corollary 17 with $\sigma=\sigma(X)$ and conclude that

$$
\lambda\left(\sigma_{1}+\sigma_{2}\right) \leq \chi_{h}(X)=\chi_{h}\left(N_{1}\right)+\chi_{h}\left(N_{2}\right)=\lambda\left(\sigma_{1}\right)+\lambda\left(\sigma_{2}\right) .
$$

We now proceed to list the smallest upper bounds on $\lambda(\sigma)$ currently known to the authors. We begin by first finding parameters $g, p, v, u_{1}, u_{2}$ and $t$ in Examples 11 and 12 that yield 4-manifolds with small $\chi_{h}$ values. By Rohlin's theorem, these 4-manifolds are nonspin if their signatures are not divisible by 16 . Unfortunately, given an integer $\sigma \geq 0$, there is no clear pattern as to which family or parameters

\begin{tabular}{|c|c|c|c|c|c|}
\hline$\sigma$ & $\lambda(\sigma) \leq$ & $X$ & $\sigma$ & $\lambda(\sigma) \leq$ & $X$ \\
\hline $0-1$ & 25 & $Q_{9}^{1}\left(W_{1,1}^{3,2}\right)$ & 50 & 86 & $P_{19}^{1}\left(W_{2,1}^{5,1}\right)$ \\
\hline 2 & 24 & $Q_{9}^{1}\left(W_{1,1}^{3,2}\right)$ & 51 & 111 & $P_{19}^{1}\left(W_{2,1}^{5,1}\right) \# \varphi Q_{9}^{1}\left(W_{1,1}^{3,2}\right)$ \\
\hline 3 & 27 & $P_{9}^{1}\left(W_{1,1}^{3,2}\right)$ & 52 & 110 & $P_{19}^{1}\left(W_{2,1}^{5,1}\right) \# \varphi Q_{9}^{1}\left(W_{1,1}^{3,2}\right)$ \\
\hline 4 & 26 & $P_{9}^{1}\left(W_{1,1}^{3,2}\right)$ & 53 & 113 & $P_{19}^{1}\left(W_{2,1}^{5,1}\right) \# \varphi P_{9}^{1}\left(W_{1,1}^{3,2}\right)$ \\
\hline 5 & 47 & $Q_{15}^{1}\left(W_{1,2}^{3,2}\right)$ & 54 & 112 & $P_{19}^{1}\left(W_{2,1}^{5,1}\right) \# \varphi P_{9}^{1}\left(W_{1,1}^{3,2}\right)$ \\
\hline 6 & 46 & $Q_{15}^{1}\left(W_{1,2}^{3,2}\right)$ & 55 & 133 & $P_{19}^{1}\left(W_{2,1}^{5,1}\right) \# \varphi Q_{15}^{1}\left(W_{1,2}^{3,2}\right)$ \\
\hline 7 & 49 & $P_{15}^{1}\left(W_{1,2}^{3,2}\right)$ & 56 & 132 & $P_{19}^{1}\left(W_{2,1}^{5,1}\right) \# \varphi Q_{15}^{1}\left(W_{1,2}^{3,2}\right)$ \\
\hline 8 & 48 & $P_{15}^{1}\left(W_{1,2}^{3,2}\right)$ & 57 & 135 & $P_{19}^{1}\left(W_{2,1}^{5,1}\right) \# \varphi P_{15}^{1}\left(W_{1,2}^{3,2}\right)$ \\
\hline $9-13$ & 59 & $Q_{18}^{1}\left(W_{1,1}^{5,1}\right)$ & 58 & 134 & $P_{19}^{1}\left(W_{2,1}^{5,1}\right) \# \varphi P_{15}^{1}\left(W_{1,2}^{3,2}\right)$ \\
\hline $14-21$ & 58 & $Q_{18}^{1}\left(W_{1,1}^{5,1}\right)$ & $59-61$ & 143 & $Q_{19}^{1}\left(W_{2,1}^{5,1}\right) \# \varphi Q_{18}^{1}\left(W_{1,1}^{5,1}\right)$ \\
\hline 22 & 57 & $Q_{18}^{1}\left(W_{1,1}^{5,1}\right)$ & $62-69$ & 142 & $Q_{19}^{1}\left(W_{2,1}^{5,1}\right) \# \varphi Q_{18}^{1}\left(W_{1,1}^{5,1}\right)$ \\
\hline 23 & 60 & $P_{18}^{1}\left(W_{1,1}^{5,1}\right)$ & 70 & 141 & $Q_{19}^{1}\left(W_{2,1}^{5,1}\right) \# \varphi Q_{18}^{1}\left(W_{1,1}^{5,1}\right)$ \\
\hline 24 & 59 & $P_{18}^{1}\left(W_{1,1}^{5,1}\right)$ & 71 & 144 & $Q_{36}^{1}\left(W_{3,1}^{5,1}\right)$ \\
\hline 25 & 84 & $P_{18}^{1}\left(W_{1,1}^{5,1}\right) \# \varphi Q_{9}^{1}\left(W_{1,1}^{3,2}\right)$ & 72 & 143 & $Q_{36}^{1}\left(W_{3,1}^{5,1}\right)$ \\
\hline 26 & 83 & $P_{18}^{1}\left(W_{1,1}^{5,1}\right) \# \varphi Q_{9}^{1}\left(W_{1,1}^{3,2}\right)$ & 73 & 146 & $P_{36}^{1}\left(W_{3,1}^{5,1}\right)$ \\
\hline 27 & 86 & $P_{18}^{1}\left(W_{1,1}^{5,1}\right) \# \varphi P_{9}^{1}\left(W_{1,1}^{3,2}\right)$ & 74 & 145 & $P_{36}^{1}\left(W_{3,1}^{5,1}\right)$ \\
\hline 28 & 85 & $P_{18}^{1}\left(W_{1,1}^{5,1}\right) \# \varphi P_{9}^{1}\left(W_{1,1}^{3,2}\right)$ & $75-81$ & 167 & $Q_{34}^{1}\left(W_{2,2}^{5,1}\right)$ \\
\hline $29-31$ & 87 & $Q_{19}^{1}\left(W_{2,1}^{5,1}\right)$ & $82-89$ & 166 & $Q_{34}^{1}\left(W_{2,2}^{5,1}\right)$ \\
\hline $32-39$ & 86 & $Q_{19}^{1}\left(W_{2,1}^{5,1}\right)$ & $90-97$ & 165 & $Q_{34}^{1}\left(W_{2,2}^{5,1}\right)$ \\
\hline $40-47$ & 85 & $Q_{19}^{1}\left(W_{2,1}^{5,1}\right)$ & 98 & 164 & $Q_{34}^{1}\left(W_{2,2}^{5,1}\right)$ \\
\hline 48 & 84 & $Q_{19}^{1}\left(W_{2,1}^{5,1}\right)$ & 99 & 167 & $P_{34}^{1}\left(W_{2,2}^{5,1}\right)$ \\
\hline 49 & 87 & $P_{19}^{1}\left(W_{2,1}^{5,1}\right)$ & 100 & 166 & $P_{34}^{1}\left(W_{2,2}^{5,1}\right)$ \\
\hline
\end{tabular}

Table 2. Upper bounds on $\lambda(\sigma)$. 
will yield a simply connected nonspin 4-manifold $X$ with $\sigma(X) \geq \sigma$ having the smallest $\chi_{h}(X)+\ell(\sigma)+1$. Hence we had to resort to a computer search.

Table 2 on the previous page lists some of the smallest upper bounds on $\lambda(\sigma)$ that we found. For example, when $\sigma=10$, Table 2 says that $\lambda(10) \leq 59$, that is, for each odd integer $k \geq 2 \cdot 59-1=117$, there exist an infinite family of pairwise nondiffeomorphic irreducible symplectic 4-manifolds and an infinite family of pairwise nondiffeomorphic irreducible nonsymplectic 4-manifolds, all of which are homeomorphic to $k \mathbb{C P} \mathbb{P}^{2} \#(k-10) \overline{\mathbb{C P}}^{2}$. The third column in Table 2 lists the simply connected 4-manifold $X$ that was used to obtain the upper bound via Corollary 17. The \# $\varphi$ symbol denotes a generalized fiber sum along the tori $T_{j}$ and/or $T_{j}^{\prime}$. We have compiled upper bounds on $\lambda(\sigma)$ for $\sigma$ up to about $1,000,000$ but we will only list a small sample here.

\section{Acknowledgements}

The authors thank Vincent Chan, David McKinnon and Shigeru Takamura for very helpful comments. We also thank the referee for suggesting numerous ways to improve the paper. Some of the computations for Table 2 were done with the aid of Maple and Mathematica.

\section{References}

[Akhmedov and Park 2008] A. Akhmedov and B. D. Park, "New symplectic 4-manifolds with nonnegative signature", J. Gökova Geom. Topol. GGT 2 (2008), 1-13. MR 2009m:57044 Zbl 1184.57017

[Akhmedov and Park 2010a] A. Akhmedov and B. D. Park, "Exotic smooth structures on small 4-manifolds with odd signatures", Invent. Math. 181:3 (2010), 577-603. MR 2012b:57048 Zbl 1206.57029

[Akhmedov and Park 2010b] A. Akhmedov and B. D. Park, "Geography of simply connected spin symplectic 4-manifolds", Math. Res. Lett. 17:3 (2010), 483-492. MR 2011h:57042 Zbl 05937386

[Akhmedov et al. 2008] A. Akhmedov, R. İ. Baykur, and B. D. Park, "Constructing infinitely many smooth structures on small 4-manifolds", J. Topol. 1:2 (2008), 409-428. MR 2010c:57040 Zbl 1146.57041

[Akhmedov et al. 2010a] A. Akhmedov, S. Baldridge, R. İ. Baykur, P. Kirk, and B. D. Park, "Simply connected minimal symplectic 4-manifolds with signature less than -1", J. Eur. Math. Soc. (JEMS) 12:1 (2010), 133-161. MR 2011b:57032 Zbl 1185.57023

[Akhmedov et al. 2010b] A. Akhmedov, B. D. Park, and G. Urzúa, "Spin symplectic 4-manifolds near Bogomolov-Miyaoka-Yau line”, J. Gökova Geom. Topol. GGT 4 (2010), 55-66. MR 2755993

[Chen 1991] Z. J. Chen, "The existence of algebraic surfaces with preassigned Chern numbers", Math. Z. 206:2 (1991), 241-254. MR 92a:14036 Zbl 0695.14018

[Donaldson 1983] S. K. Donaldson, "An application of gauge theory to four-dimensional topology", J. Differential Geom. 18:2 (1983), 279-315. MR 85c:57015 Zbl 0507.57010

[Donaldson 1996] S. K. Donaldson, "The Seiberg-Witten equations and 4-manifold topology", Bull. Amer. Math. Soc. (N.S.) 33:1 (1996), 45-70. MR 96k:57033 Zbl 0872.57023 
[Fintushel and Stern 1998] R. Fintushel and R. J. Stern, "Knots, links, and 4-manifolds", Invent. Math. 134:2 (1998), 363-400. MR 99j:57033 Zbl 0914.57015

[Fintushel and Stern 2009] R. Fintushel and R. J. Stern, "Six lectures on four 4-manifolds", pp. 265-315 in Low dimensional topology, edited by T. S. Mrowka and P. S. Ozsváth, IAS/Park City Math. Ser. 15, Amer. Math. Soc., Providence, RI, 2009. MR 2010g:57035 Zbl 1195.57001

[Fintushel et al. 2007] R. Fintushel, B. D. Park, and R. J. Stern, "Reverse engineering small 4manifolds", Algebr. Geom. Topol. 7 (2007), 2103-2116. MR 2009h:57044 Zbl 1142.57018

[Freedman 1982] M. H. Freedman, "The topology of four-dimensional manifolds", J. Differential Geom. 17:3 (1982), 357-453. MR 84b:57006 Zbl 0528.57011

[Gompf 1995] R. E. Gompf, "A new construction of symplectic manifolds", Ann. of Math. (2) 142:3 (1995), 527-595. MR 96j:57025 Zbl 0849.53027

[Gompf and Stipsicz 1999] R. E. Gompf and A. I. Stipsicz, 4-manifolds and Kirby calculus, Graduate Studies in Mathematics 20, American Mathematical Society, Providence, RI, 1999. MR 2000h:57038 Zbl 0933.57020

[Hamilton and Kotschick 2006] M. J. D. Hamilton and D. Kotschick, "Minimality and irreducibility of symplectic four-manifolds", Int. Math. Res. Not. 2006:2 (2006), Art. ID 35032, 13. MR 2007i:57023 Zbl 1101.53052

[Harris and Morrison 1998] J. Harris and I. Morrison, Moduli of curves, Graduate Texts in Mathematics 187, Springer, New York, 1998. MR 99g:14031 Zbl 0913.14005

[Kotschick 1997] D. Kotschick, "The Seiberg-Witten invariants of symplectic four-manifolds (after C. H. Taubes)", pp. 195-220 in Séminaire Bourbaki 1995/1996 (Exposé 812), Astérisque 241, Société Mathématique de France, Paris, 1997. MR 98h:57057 Zbl 0882.57026

[Li 1999] T.-J. Li, "Smoothly embedded spheres in symplectic 4-manifolds", Proc. Amer. Math. Soc. 127:2 (1999), 609-613. MR 99c:57055 Zbl 0911.57018

[Li and Stipsicz 2002] T.-J. Li and A. I. Stipsicz, "Minimality of certain normal connected sums", Turkish J. Math. 26:1 (2002), 75-80. MR 2003c:57028 Zbl 1002.57047

[Luo 2000] F. Luo, "Torsion elements in the mapping class group of a surface", preprint, 2000. arXiv 0004048

[McCarthy and Wolfson 1994] J. D. McCarthy and J. G. Wolfson, "Symplectic normal connect sum", Topology 33:4 (1994), 729-764. MR 95h:57038 Zbl 0812.53033

[Morgan et al. 1997] J. W. Morgan, T. S. Mrowka, and Z. Szabó, "Product formulas along $T^{3}$ for Seiberg-Witten invariants”, Math. Res. Lett. 4:6 (1997), 915-929. MR 99f:57039 Zbl 0892.57021

[Némethi 1999] A. Némethi, "Five lectures on normal surface singularities", pp. 269-351 in Low dimensional topology (Eger, 1996/Budapest, 1998), edited by K. Böröczky, Jr. et al., Bolyai Soc. Math. Stud. 8, János Bolyai Math. Soc., Budapest, 1999. MR 2001g:32066 Zbl 0958.32026

[Nielsen 1937] J. Nielsen, "Die Struktur periodischer Transformationen von Flächen”, Danske Vid. Selsk. Mat.-Fys. Medd. 15:1 (1937), 1-77. Zbl 0017.13302

[Niepel 2005] M. Niepel, "Examples of symplectic 4-manifolds with positive signature", pp. 235-242 in Geometry and topology of manifolds, edited by H. U. Boden et al., Fields Inst. Commun. 47, Amer. Math. Soc., Providence, RI, 2005. MR 2006k:57073 Zbl 1095.57024

[Park 2002] J. Park, “The geography of spin symplectic 4-manifolds”, Math. Z. 240:2 (2002), 405421. MR 2003c:57030 Zbl 1030.57032

[Park 2003] J. Park, "Exotic smooth structures on 4-manifolds, II", Topology Appl. 132:2 (2003), 195-202. MR 2004d:57033 Zbl 1028.57032

[Park and Szabó 2000] B. D. Park and Z. Szabó, "The geography problem for irreducible spin fourmanifolds”, Trans. Amer. Math. Soc. 352:8 (2000), 3639-3650. MR 2000m:57037 Zbl 0947.57023 
[Persson 1981] U. Persson, "Chern invariants of surfaces of general type", Compositio Math. 43:1 (1981), 3-58. MR 83b:14012 Zbl 0479.14018

[Rohlin 1952] V. A. Rohlin, "New results in the theory of four-dimensional manifolds", Doklady Akad. Nauk SSSR (N.S.) 84 (1952), 221-224. In Russian. MR 14,573b Zbl 0046.40702

[Stipsicz 1998] A. I. Stipsicz, "Simply connected 4-manifolds near the Bogomolov-Miyaoka-Yau line”, Math. Res. Lett. 5:6 (1998), 723-730. MR 2000h:57047 Zbl 0947.57032

[Stipsicz 1999] A. I. Stipsicz, "Simply connected symplectic 4-manifolds with positive signature", Turkish J. Math. 23:1 (1999), 145-150. MR 2001e:57029 Zbl 0945.57010

[Stipsicz 2000] A. I. Stipsicz, "Chern numbers of certain Lefschetz fibrations", Proc. Amer. Math. Soc. 128:6 (2000), 1845-1851. MR 2000j:57062 Zbl 0982.57012

[Szabó 1998] Z. Szabó, "Simply-connected irreducible 4-manifolds with no symplectic structures", Invent. Math. 132:3 (1998), 457-466. MR 99f:57033 Zbl 0906.57014

[Takamura 2004] S. Takamura, "Towards the classification of atoms of degenerations, I: Splitting criteria via configurations of singular fibers", J. Math. Soc. Japan 56:1 (2004), 115-145. MR 2005c:14009 Zbl 1054.14016

[Taubes 1994] C. H. Taubes, "The Seiberg-Witten invariants and symplectic forms", Math. Res. Lett. 1:6 (1994), 809-822. MR 95j:57039 Zbl 0853.57019

[Taubes 1996] C. H. Taubes, "SW $\Rightarrow$ Gr: From the Seiberg-Witten equations to pseudo-holomorphic curves”, J. Amer. Math. Soc. 9:3 (1996), 845-918. MR 97a:57033 Zbl 0867.53025

[Usher 2006] M. Usher, "Minimality and symplectic sums", Int. Math. Res. Not. 2006:16 (2006), Art. ID 49857, 17. MR 2007h:53139 Zbl 1110.57017

[Witten 1994] E. Witten, "Monopoles and four-manifolds", Math. Res. Lett. 1:6 (1994), 769-796. MR 96d:57035 Zbl 0867.57029

Received March 6, 2011. Revised January 9, 2013.

ANAR AKHMEDOV

SCHOOL OF MATHEMATICS

UNIVERSITY OF MINNESOTA

MiNNEAPOLIS, MN 55455

UNITED STATES

akhmedov@math.umn.edu

MARK C. Hughes

DEPARTMENT OF MATHEMATICS

STONY BROOK UNIVERSITY

STONY BROOK, NY 11794-3651

UNITED STATES

hughes@math.sunysb.edu

B. DOUG PARK

Department of Pure Mathematics

UNIVERSITY OF WATERLOO

WATERLOO, ON

N2L 3G1

CANADA

bdpark@uwaterloo.ca 


\title{
PACIFIC JOURNAL OF MATHEMATICS
}

\author{
msp.org/pjm
}

Founded in 1951 by E. F. Beckenbach (1906-1982) and F. Wolf (1904-1989)

\section{EDITORS}

V. S. Varadarajan (Managing Editor)

Department of Mathematics

University of California

Los Angeles, CA 90095-1555

pacific@math.ucla.edu

Paul Balmer

Department of Mathematics

University of California

Los Angeles, CA 90095-1555

balmer@math.ucla.edu

Daryl Cooper

Department of Mathematics

University of California

Santa Barbara, CA 93106-3080 cooper@math.ucsb.edu

Jiang-Hua $\mathrm{Lu}$

Department of Mathematics

The University of Hong Kong

Pokfulam Rd., Hong Kong jhlu@maths.hku.hk
Don Blasius

Department of Mathematics University of California

Los Angeles, CA 90095-1555

blasius@math.ucla.edu

Robert Finn

Department of Mathematics Stanford University

Stanford, CA 94305-2125

finn@math.stanford.edu

Sorin Popa

Department of Mathematics

University of California

Los Angeles, CA 90095-1555

popa@math.ucla.edu

Paul Yang

Department of Mathematics

Princeton University

Princeton NJ 08544-1000

yang@math.princeton.edu

\section{PRODUCTION}

Silvio Levy, Scientific Editor, production@msp.org

\section{SUPPORTING INSTITUTIONS}

ACADEMIA SINICA, TAIPEI

CALIFORNIA INST. OF TECHNOLOGY

INST. DE MATEMÁTICA PURA E APLICADA

KEIO UNIVERSITY

MATH. SCIENCES RESEARCH INSTITUTE

NEW MEXICO STATE UNIV.

OREGON STATE UNIV.

\author{
STANFORD UNIVERSITY \\ UNIV. OF BRITISH COLUMBIA \\ UNIV. OF CALIFORNIA, BERKELEY \\ UNIV. OF CALIFORNIA, DAVIS \\ UNIV. OF CALIFORNIA, LOS ANGELES \\ UNIV. OF CALIFORNIA, RIVERSIDE \\ UNIV. OF CALIFORNIA, SAN DIEGO \\ UNIV. OF CALIF., SANTA BARBARA
}

\author{
Vyjayanthi Chari \\ Department of Mathematics \\ University of California \\ Riverside, CA 92521-0135 \\ chari@math.ucr.edu \\ Kefeng Liu \\ Department of Mathematics \\ University of California \\ Los Angeles, CA 90095-1555 \\ liu@math.ucla.edu \\ Jie Qing \\ Department of Mathematics \\ University of California \\ Santa Cruz, CA 95064 \\ qing@cats.ucsc.edu
}

These supporting institutions contribute to the cost of publication of this Journal, but they are not owners or publishers and have no responsibility for its contents or policies.

See inside back cover or msp.org/pjm for submission instructions.

The subscription price for 2013 is US \$400/year for the electronic version, and \$485/year for print and electronic.

Subscriptions, requests for back issues and changes of subscribers address should be sent to Pacific Journal of Mathematics, P.O. Box 4163, Berkeley, CA 94704-0163, U.S.A. The Pacific Journal of Mathematics is indexed by Mathematical Reviews, Zentralblatt MATH, PASCAL CNRS Index, Referativnyi Zhurnal, Current Mathematical Publications and the Science Citation Index.

The Pacific Journal of Mathematics (ISSN 0030-8730) at the University of California, c/o Department of Mathematics, 798 Evans Hall \#3840, Berkeley, CA 94720-3840, is published monthly except July and August. Periodical rate postage paid at Berkeley, CA 94704, and additional mailing offices. POSTMASTER: send address changes to Pacific Journal of Mathematics, P.O. Box 4163, Berkeley, CA 94704-0163.

PJM peer review and production are managed by EditFLOW ${ }^{\circledR}$ from Mathematical Sciences Publishers.

PUBLISHED BY

mathematical sciences publishers

nonprofit scientific publishing

http://msp.org/

(C) 2013 Mathematical Sciences Publishers 


\section{PACIFIC JOURNAL OF MATHEMATICS}

Volume $261 \quad$ No. $2 \quad$ February 2013

Geography of simply connected nonspin symplectic 4-manifolds with positive signature

Anar Akhmedov, Mark C. Hughes and B. Doug Park

Schur-Horn theorems in $\mathrm{II}_{\infty}$-factors

283

MARTÍN ARGERAMI and PEDRo MASSEY

Classification of positive solutions for an elliptic system with a higher-order

fractional Laplacian

JiNGBo DOU and CHANGZHENG QU

Bound states of asymptotically linear Schrödinger equations with compactly supported potentials

MingWEN FEI and HUICHENG YiN

Type I almost homogeneous manifolds of cohomogeneity one, III

DANIEL GUAN

The subrepresentation theorem for automorphic representations

MARCELA HANZER

Variational characterizations of the total scalar curvature and eigenvalues of the

Laplacian

Seungsu Hwang, JeOngwook Chang and GabJin Yun

Fill-ins of nonnegative scalar curvature, static metrics, and quasi-local mass

JEFFREY L. JAUREGUI

Operator algebras and conjugacy problem for the pseudo-Anosov automorphisms of a surface

\section{IGOR NIKOLAEV}

Connected sums of closed Riemannian manifolds and fourth-order conformal invariants

DAVID RASKE

Ruled minimal surfaces in the three-dimensional Heisenberg group

Heayong Shin, Young Wook Kim, Sung-Eun Koh, Hyung Yong LeE and SEONG-DEOG YANG

$G$-bundles over elliptic curves for non-simply laced Lie groups and configurations of lines in rational surfaces

MANG XU and JIAJIN ZHANG 